\title{
ARTICLE
}

\section{Magnesium isoglycyrrhizinate ameliorates high fructose- induced liver fibrosis in rat by increasing miR-375-3p to suppress JAK2/STAT3 pathway and TGF- $\beta 1 /$ Smad signaling}

\author{
Yan-zi Yang ${ }^{1}$, Xiao-juan Zhao', Hong-jiang Xu' ${ }^{2}$, Shan-chun Wang ${ }^{2}$, Ying Pan ${ }^{1}$, Shui-juan Wang ${ }^{1}$, Qiang Xu ${ }^{1}$, Rui-qing Jiao ${ }^{1}$, \\ Hong-mei $\mathrm{Gu}^{2}$ and Ling-dong Kong ${ }^{1}$
}

\begin{abstract}
Increasing evidence has demonstrated that excessive fructose intake induces liver fibrosis. Epithelial-mesenchymal transition (EMT) driven by transforming growth factor- $\beta 1$ (TGF- $\beta 1$ )/mothers against decapentaplegic homolog (Smad) signaling activation promotes the occurrence and development of liver fibrosis. Magnesium isoglycyrrhizinate is clinically used as a hepatoprotective agent to treat liver fibrosis, but its underlying molecular mechanism has not been identified. Using a rat model, we found that high fructose intake reduced microRNA (miR)-375-3p expression and activated the janus-activating kinase 2 (JAK2)/signal transducer and activator of transcription 3 (STAT3) cascade and TGF- $\beta 1 /$ Smad signaling, which is consistent with the EMT and liver fibrosis. To further verify these observations, BRL-3A cells and/or primary rat hepatocytes were exposed to high fructose and/or transfected with a miR-375-3p mimic or inhibitor or treated with a JAK2 inhibitor, and we found that the low expression of miR-375-3p could induce the JAK2/STAT3 pathway to activate TGF- $\beta 1 /$ Smad signaling and promote the EMT. Magnesium isoglycyrrhizinate was found to ameliorate high fructose-induced EMT and liver fibrosis in rats. More importantly, magnesium isoglycyrrhizinate increased miR$375-3 p$ expression to suppress the JAK2/STAT3 pathway and TGF- $\beta 1 /$ Smad signaling in these animal and cell models. This study provides evidence showing that magnesium isoglycyrrhizinate attenuates liver fibrosis associated with a high fructose diet.
\end{abstract}

Keywords: magnesium isoglycyrrhizinate; excessive fructose intake; EMT; MicroRNA-375-3p; JAK2/STAT3 pathway; TGF- $\beta 1 / S m a d$ signaling

Acta Pharmacologica Sinica (2019) 40:879-894; https://doi.org/10.1038/s41401-018-0194-4

\section{INTRODUCTION}

Liver fibrosis is a wound-healing response to chronic liver injury [1]. Transforming growth factor- $\beta 1$ (TGF- $\beta 1$ ) is recognized as a major profibrogenic cytokine in hepatocytes that play a crucial role in the epithelial-mesenchymal transition (EMT) primarily via a canonical mothers against decapentaplegic homolog (Smad)dependent mechanism [2, 3]. In liver fibrosis, janus-activating kinase 2 (JAK2) expression and activity are increased in experimental rodents and humans $[4,5]$. Signal transducer and activator of transcription 3 (STAT3) enhances hepatic fibrosis by upregulating TGF- $\beta 1$ in primary hepatocytes and human hepatocarcinoma cells (HepG2) in response to interleukin-6 [6]. Excessive fructose consumption is a risk factor for liver fibrosis in patients and animal models $[7,8]$. High fructose feeding is reported to activate the liver JAK2/STAT3 pathway in rats [9]. Thus, an interaction may occur between the JAK2/STAT3 pathway and TGF- $\beta 1 /$ Smad signaling in high fructose-induced EMT in liver fibrosis [3]. MicroRNAs (miRNAs) function as pro-fibrotic or antifibrotic mediators in liver fibrosis [10-13]. MiR-375-3p expression is monotonically decreased in liver biopsy tissues from class III obese human subjects [14]. According to the TargetScan database and recent studies, putative miR-375-3p binding sites are located on the $3^{\prime}$ untranslated region (UTR) of both human and rat JAK2 messenger RNA (mRNA) $[15,16]$. The loss of miR-375-3p expression enhances the persistent activation of the JAK2/STAT3 pathway in gastric epithelial cells stimulated by lipopolysaccharide [17]. We hypothesized that high fructose consumption decreases miR-375-3p expression to activate the JAK2/STAT3 pathway and TGF- $\beta 1 / \mathrm{Smad}$ signaling and promote the EMT in liver fibrosis.

Currently, no specific efficacious therapy is available for the treatment of liver fibrosis because the altered modulation of the EMT sustains liver fibrogenesis [18]. Pioglitazone is a peroxisome proliferator-activated receptor- $\gamma$ (PPAR- $\gamma$ ) agonist that improves liver histology and fibrosis in patients with nonalcoholic fatty liver disease $[19,20]$. However, long-term treatment appears to increase the risk of bladder cancer among people with type 2 diabetes [21]. Magnesium isoglycyrrhizinate, which is a magnesium salt with an 18-a glycyrrhizic acid stereoisomer, has antiinflammation and hepatoprotection activities. Magnesium isoglycyrrhizinate is clinically used to treat chronic liver disease with few side effects [22-26]. Magnesium isoglycyrrhizinate can decrease the blood hepatic fibrosis indicators hyaluronic acid and TGF- $\beta 1$ in

\footnotetext{
${ }^{1}$ State Key Laboratory of Pharmaceutical Biotechnology, School of Life Sciences, Nanjing University, Nanjing 210023, China and ${ }^{2}$ Jiangsu Key Laboratory of Targeted Antiviral Research, Chia Tai Tianqing Pharmaceutical Group Co., Ltd, Nanjing 210023, China

Correspondence: Hong-mei Gu (ghm@cttq.com) or Ling-dong Kong (kongld@nju.edu.cn)

These authors contributed equally: Yan-zi Yang, Xiao-juan Zhao.
}

Received: 30 May 2018 Accepted: 8 November 2018

Published online: 19 December 2018 
880

patients with chronic type B hepatitis [27]. Magnesium isoglycyrrhizinate also ameliorates carbon tetrachloride-induced liver fibrosis in rats with the reduction of TGF- $\beta 1$ and phosphorylated (p)-Smad3 protein levels $[28,29]$. Our previous study showed that magnesium isoglycyrrhizinate relieves excessive fructose-induced lipid metabolism disorder and inflammation in rats by blocking inflammatory signaling activation [30]. However, the molecular mechanisms by which magnesium isoglycyrrhizinate alleviates high fructose-induced liver fibrosis are largely unknown.

First, this study demonstrated that low miR-375-3p expression facilitated the JAK2/STAT3 pathway expression and TGF- $\beta 1 / \mathrm{Smad}$ signaling activation, causing the EMT in high fructose-induced liver fibrosis. Second, magnesium isoglycyrrhizinate was found to alleviate the EMT in high fructose-induced liver fibrosis potentially by upregulating miR-375-3p expression to inhibit the JAK2/STAT3 pathway and TGF- $\beta 1 /$ Smad signaling activation. These results provide evidence that magnesium isoglycyrrhizinate-mediated high miR-375-3p expression could be a therapeutic strategy for the treatment of liver fibrosis associated with excessive fructose consumption.

\section{MATERIALS AND METHODS}

Reagents and antibodies

Fructose crystallization for animal experiments was purchased from Shandong Xiwang Sager Industry Co., Ltd. (Binzhou, China). Magnesium isoglycyrrhizinate injections for rats and powder for the cell experiments were provided by Chia Tai Tianqing Pharmaceutical Group Co., Ltd (Nanjing, China, Lot. number: 215020, 150819104, and 150829204). The pioglitazone tablets were purchased from Jiangsu Deyuan Pharmaceutical Co., Ltd (Lianyungang, China, Lot. number: 150792). Fructose and pioglitazone for the cell experiments were purchased from SigmaAldrich Inc. (St. Louis, MO, USA). The alanine aminotransferase (ALT), aspartate aminotransferase (AST), and hydroxyproline and hyaluronic acid assay kits were purchased from Jiancheng Biotechnology Co., Ltd (Nanjing, China). Fetal bovine serum (FBS) was purchased from Wisent Technology (St-Bruno, QC, Canada). Dulbecco's modified Eagle's medium (DMEM) was purchased from Yuanpei Biological Technology Co., Ltd (Shanghai, China). Invitrogen ${ }^{T M}$ TRlzol reagent was got from Thermo Fisher Scientific (Shanghai, China). Reverse transcription system kit, HiScribe reverse transcriptase, dNTPs, RNase inhibitor, and ChamQ SYBR qPCR master mix were obtained from Vazyme Biotechnology Co., Ltd (Nanjing, China), respectively. Lipofectamine 2000 was purchased from Invitrogen (Carlsbad, CA, USA). The JAK2 inhibitor AG490 was purchased from Medchem Express Co., Ltd (Princeton, NJ, USA). The miR-375-3p mimic, miR-375-3p inhibitor, the respective negative control, and the pmirGLO reporter vector were synthesized by GenePharma (Shanghai, China). The Dual Luciferase assay kit was purchased from Promega (Madison, IL, USA). The cell lysis buffer was purchased from Beyotime Biotechnology (P0013, Nanjing, China). The rabbit anti-TGF- $\beta 1$ (V: sc-146), goat anti-Smad2 (S-20: sc-6200), rabbit anti-p-Smad2 (Ser467: sc-101801), mouse anti-Smad3 (38-Q: sc-101154), rabbit anti-p-Smad3 (Ser208: sc-130218), mouse anti-Smad4 (B-8: sc7966), and rabbit anti-GAPDH (sc-25778) antibodies were purchased from Santa Cruz Biotechnology Co., Ltd (Santa Cruz, CA, USA). The rabbit anti-Collagen 1 (ab34710), rabbit anti-fibronectin (ab2413), rabbit anti-a smooth muscle actin (a-SMA) (ab5694), rabbit anti-N-Cadherin (ab18203), rabbit anti-STAT3 (ab68153), and rabbit anti-p-STAT3 (ab76315) antibodies were purchased from Abcam (Cambridge, MA, USA). The mouse anti-E-Cadherin (4A2), rabbit anti-vimentin (D21H3), rabbit anti-JAK2 (D2E12), and rabbit anti-p-JAK2 (C80C3) antibodies and horseradish peroxidase (HRP)-conjugated rabbit anti-lgG (AP132P) were obtained from Cell Signaling Technology (Cambridge, MA, USA). The mouse anti$\beta$-Actin (ABM-0001) antibody was purchased from Zoonbio
Biotechnology Co., Ltd (Nanjing, China). HRP-conjugated mouse anti-lgG (HAF007) and HRP-conjugated goat anti-lgG (HAF017) were obtained from R\&D Systems (Minneapolis, MN, USA). Alexa Fluor 488 goat anti-rabbit lgG (A11008) and Alexa Fluor 555 goat anti-mouse IgG (A21422) were purchased from Life Technology (Gaithersburg, MD, USA).

\section{Animals and treatment}

Male Sprague-Dawley rats aged 6-7 weeks (180-220 g) were purchased from the Experimental Animal Center of Zhejiang Province (Hangzhou, China; Production license: SCXK 2014-0001). The rats were maintained under a controlled temperature $(22 \pm$ $\left.2{ }^{\circ} \mathrm{C}\right)$, humidity $(55 \% \pm 5 \%)$, and 12 -h light/12-h dark cycle with the lights on from 9:00 a.m. to 9:00 p.m. The rats fed a standard chow diet and water ad libitum for 1 week of acclimatization before the experiment. Each rat was given drinking water or $100 \mathrm{~mL}$ drinking water containing $10 \%$ fructose (wt/vol) every day for 17 weeks. Starting during the 7th week, the rats in the normal control group as well as the fructose control group received saline intraperitoneally (i.p.) for the following 11 weeks, while the rats in the other three groups received 10,20 , and $40 \mathrm{mg} / \mathrm{kg}$ magnesium isoglycyrrhizinate injection i.p., and the final group received a $4 \mathrm{mg} / \mathrm{kg}$ pioglitazone tablet suspension intragastrically $(n=10$ / group). The body weights were recorded weekly. The doses of magnesium isoglycyrrhizinate and pioglitazone were selected based on conversion from human clinical trials and preliminary studies [31-34]. All animal experimental protocols were approved by the Institutional Animal Care and Use Committee of Nanjing University. The animal welfare and experimental procedures were carried out in accordance with the criteria outlined in the "Guide for the Care and Use of Laboratory Animals" enacted by the National Academy of Sciences, and published by the National Institutes of Health (NIH publication 86-23 revised 1985) and related ethnical regulations of Nanjing University (SYXK (SU) 20090017). All efforts were made to minimize the rats' suffering and reduce the number of rats used.

Urine, blood, and tissue collection

Each rat was placed in a metabolic cage with free access to standard diet and water. Urine samples were collected for $24 \mathrm{~h}$. At the end of the animal experiments, the rats were anesthetized via an i.p. injection of $50 \mathrm{mg} / \mathrm{kg}$ sodium pentobarbital. The blood samples were collected by using carotid artery intubation and then centrifuged at $3000 \times g$ for $10 \mathrm{~min}\left(4^{\circ} \mathrm{C}\right)$ to obtain the serum. These urine and serum samples were stored at $-80^{\circ} \mathrm{C}$ for the biochemical assays. The liver tissue samples were quickly dissected on ice and weighed. Some liver samples were immediately fixed for the histological studies, while the other samples were stored at $-80^{\circ} \mathrm{C}$ for the analysis of biochemical indexes, quantitative real-time PCR (qRT-PCR) and Western blot.

\section{Liver histopathology}

Standardized protocols were used to observe the liver histological changes. Formalin-fixed liver specimens were stained with hematoxylin and eosin (H\&E) reagent, Masson trichrome, and Sirius red solution, and then analyzed.

Biochemical analysis

The serum ALT, AST, and hyaluronic acid levels and urine hydroxyproline levels were determined with standard diagnostic kits, according to the manufacturer's instructions.

Immunohistofluorescence

The rat liver tissues were fixed with $4 \%$ paraformaldehyde and embedded in paraffin. Subsequently, these paraffin sections were deparaffinized with xylene and rehydrated with graded ethanol. Then, the sections were blocked with $5 \%$ normal goat serum for $1 \mathrm{~h}$ and incubated with primary antibodies at $4{ }^{\circ} \mathrm{C}$ overnight. After 
being washed with $0.01 \mathrm{M}$ phosphate-buffered solution (PBS) three times, the sections were incubated with fluorophoreconjugated secondary antibodies for $60 \mathrm{~min}$ at room temperature, and then stained with $4^{\prime}, 6$-diamidino-2-phenylindole (DAPI) for $15 \mathrm{~min}$. These sections were examined under a confocal laserscanning microscope (TCS SP8-MaiTai MP; Leica, Wetzlar, Germany). The antibodies used for the immunohistofluorescence analysis included mouse anti-E-Cadherin (dilution 1:50), rabbit anti-N-Cadherin (dilution 1:200), Alexa Fluor 555 goat anti-mouse IgG (dilution 1:500), and Alexa Fluor 488 goat anti-rabbit IgG (dilution 1:500).

\section{Immunohistochemistry}

The rat liver tissues were fixed with $4 \%$ paraformaldehyde and embedded in paraffin. Sections were deparaffinized in xylene and rehydrated with graded ethanol, and then treated with $1 \% \mathrm{H}_{2} \mathrm{O}_{2}$ for $15 \mathrm{~min}$. These sections were blocked with bovine serum albumin and incubated with primary antibodies at $4{ }^{\circ} \mathrm{C}$ overnight. After being washed with PBS, the sections were incubated with a biotinylated secondary antibody and an avidin-biotin peroxidase complex. Then, the slides were counterstained with hematoxylin. Rabbit anti-vimentin (dilution 1:100) was used for the immunohistochemistry.

Cell culture

Cell line of Buffalo rat liver cells (BRL-3A) was provided by Shanghai Institutes for Biological Sciences (Shanghai, China). Primary hepatocytes were isolated from the Sprague-Dawley rats (5-6 weeks of age) by two-step collagenase perfusion [35]. These cells were cultured in DMEM $(4.5 \mathrm{mg} / \mathrm{mL}$ glucose) supplemented with $10 \% \mathrm{FBS}$ at $37{ }^{\circ} \mathrm{C}$ in a humidified $5 \% \mathrm{CO}_{2}$ atmosphere.

The BRL-3A cells and primary rat hepatocytes were seeded in 6-well plates $\left(2 \mathrm{~mL} /\right.$ well, $1.0 \times 10^{5}$ cells $\left./ \mathrm{mL}\right)$ or 12 -well plates $(1$ $\mathrm{mL} /$ well, $1.0 \times 10^{5}$ cells $/ \mathrm{mL}$ ), cultured in DMEM (containing $10 \%$ FBS) for $12 \mathrm{~h}$. After $12 \mathrm{~h}$ of starvation in serum-free medium for synchronization, the BRL-3A cells and primary rat hepatocytes were maintained in DMEM (containing 10\% FBS) and exposed to $0.1 \%$ dimethyl sulfoxide (DMSO) alone (Normal-Control), $0.1 \%$ DMSO and $5 \mathrm{mM}$ fructose (Fructose-Vehicle), or 0.1\% DMSO and $5 \mathrm{mM}$ fructose coincubated with 10,20 , or $40 \mu \mathrm{M}$ magnesium isoglycyrrhizinate or $10 \mu \mathrm{M}$ pioglitazone. These cells were treated for $8 \mathrm{~h}$ for the detection of miR-375-3p expression via a qRT-PCR analysis, $24 \mathrm{~h}$ for the detection of the JAK2, p-JAK2, and p-STAT3 protein levels, or $48 \mathrm{~h}$ for the detection of the E-Cadherin, $\mathrm{a}-\mathrm{SMA}$, Collagen 1, fibronectin, TGF- $\beta 1$, p-Smad2, p-Smad3, and Smad4 protein levels by a Western blot analysis.

The BRL-3A cells were cultured in DMEM medium supplemented with $10 \%$ FBS. The BRL-3A cells were treated with the JAK2 inhibitor AG490 for $6 \mathrm{~h}$. Then, these cells were placed in $5 \mathrm{mM}$ fructose with $0.1 \%$ DMSO, $5 \mathrm{mM}$ fructose with $0.1 \%$ DMSO coincubated with $40 \mu \mathrm{M}$ magnesium isoglycyrrhizinate, or $10 \mu \mathrm{M}$ pioglitazone for 24 or $48 \mathrm{~h}$. The protein levels of JAK2, p-JAK2, $\mathrm{p}$ STAT3, TGF- $\beta 1$, p-Smad2, p-Smad3, Smad4, E-Cadherin, a-SMA, and Collagen 1 were detected using a Western blot analysis.

Following the instructions of Lipofectamine 2000, the BRL-3A cells were transfected with miR-375-3p mimic ( $50 \mathrm{nM})$, miR-375-3p inhibitor $(80 \mathrm{nM})$, or respective negative control. The efficiency of the 6-h miR-375-3p mimic or inhibitor transfection was evaluated by measuring miR-375-3p expression through qRT-PCR. After $6 \mathrm{~h}$ transfection, these cells were incubated in DMEM medium supplemented with $10 \% \mathrm{FBS}$ and $5 \mathrm{mM}$ fructose, in the presence or absence of $40 \mu \mathrm{M}$ magnesium isoglycyrrhizinate or $10 \mu \mathrm{M}$ pioglitazone for 24 or $48 \mathrm{~h}$, respectively. A Western blot analysis was used to detect the protein levels of JAK2, p-JAK2, p-STAT3, TGF- $\beta 1, p-S m a d 2, p-S m a d 3$, Smad4, a-SMA, Collagen 1, and ECadherin under different circumstances.

The selected concentrations and incubation time of these reagents were based on preliminary experiments and other reports $[36,37]$. The protein concentrations were determined by using a BCA protein assay kit (Pierce, Rockford, IL, USA). The total cellular proteins and RNA were extracted from the cell lysates obtained by cell lysis buffer. These samples were stored at $-80^{\circ} \mathrm{C}$ until assays.

Luciferase assay

BRL-3A cells in 24-well plates were cotransfected with $500 \mathrm{ng}$ of the pmirGLO reporter vector carrying the wild-type (WT) or mutant $3^{\prime}$-UTR site or not, and $50 \mathrm{nM}$ of the miR-375-3p mimic or normal control. After $24 \mathrm{~h}$ of transfection, the firefly luciferase activity was measured by using a Dual Luciferase assay kit and normalized to a Renilla luciferase reference plasmid.

Immunofluorescence

The tested BRL-3A cells and primary rat hepatocytes were washed with PBS three times and fixed with 4\% paraformaldehyde for $30 \mathrm{~min}$. Following three washes, these cells were blocked with immunostaining blocking buffer with $0.5 \%$ Triton X-100 for $1 \mathrm{~h}$ and incubated with the primary antibody at $4{ }^{\circ} \mathrm{C}$ overnight. After three washes with PBS, these cells were incubated with fluorophore-conjugated secondary antibodies for $60 \mathrm{~min}$ at room temperature, and then stained with DAPI for $15 \mathrm{~min}$. These cells were examined under a confocal laser-scanning microscope (TCS SP8-MaiTai MP; Leica, Wetzlar, Germany). The antibodies used for the immunofluorescence included mouse anti-E-Cadherin (dilution 1:50), rabbit anti-a-SMA (dilution 1:100), rabbit anti-Collagen 1 (dilution 1:500), Alexa Fluor 555 goat anti-mouse lgG (dilution 1:500), and Alexa Fluor 488 goat anti-rabbit IgG (dilution 1:500).

Western blot analysis

The protein samples from the rat liver tissues, cultured BRL-3A cells, and primary rat hepatocytes were resolved by $10 \%$ sodium dodecyl sulfate-polyacrylamide gel electrophoresis (SDS-PAGE) and electrophoretically transferred onto polyvinylidene fluoride membranes (Millipore, Bedford, MA, USA). The primary antibodies included rabbit anti-JAK2 (dilution 1:800), rabbit anti-p-JAK2 (dilution 1:800), rabbit anti-STAT3 (dilution 1:1000), rabbit anti-pSTAT3 (dilution 1:1000), rabbit anti-TGF- $\beta 1$ (dilution 1:1000), goat anti-Smad2 (dilution 1:1000), rabbit anti-p-Smad2 (dilution 1:1000), mouse anti-Smad3 (dilution 1:1000), rabbit anti-p-Smad3 (dilution 1:1000), mouse anti-Smad4 (dilution 1:1 000), rabbit antia-SMA (dilution 1:1000), rabbit anti-vimentin (dilution 1:1000), mouse anti-E-Cadherin (dilution 1:1000), rabbit anti-N-Cadherin (dilution 1:1000), rabbit anti-Collagen 1 (dilution 1:800), rabbit anti-fibronectin (dilution 1:1000), rabbit anti-GAPDH (dilution $1: 1000$ ), and mouse anti- $\beta$-Actin (dilution 1:3000). The blots were incubated with the primary antibodies in $5 \%$ milk at $4{ }^{\circ} \mathrm{C}$ overnight, followed by HRP-conjugated anti-rabbit IgG antibody (dilution 1:20,000), HRP-conjugated anti-mouse IgG antibody (dilution 1:2000) or HRP-conjugated anti-goat IgG antibody (dilution 1:2000). The immunoreactive bands were visualized via enhanced chemiluminescence (Cell Signaling Technology) and then quantified via densitometry using the ImageJ software (version 1.42q, National Institutes of Health).

\section{RNA isolation and qRT-PCR analysis}

The total RNA was isolated from the rat liver tissues, BRL-3A cells, and primary rat hepatocytes by TRIzol reagent according to the manufacturer's recommendations. The primers used are listed in Table 1.

miRNAs were extracted by a specific stem loop in RNA from reverse transcription. On these plates, the reverse transcription reaction mixture contained $1 \mu \mathrm{g}$ of purified total RNA, $0.75 \mu \mathrm{L}$ of $10 \mu \mathrm{M}$ stem loop RT primers, $2 \mu \mathrm{L}$ of RT buffer, $1 \mu \mathrm{L}$ of $40 \mathrm{U} / \mu \mathrm{L}$ HiScribe reverse transcriptase, $1 \mu \mathrm{L}$ of $10 \mathrm{mM}$ dNTPs, and $0.25 \mu \mathrm{L}$ of $40 \mathrm{U} / \mu \mathrm{L}$ RNase inhibitor in a volume of $10 \mu \mathrm{L}$ via the addition of $\mathrm{H}_{2} \mathrm{O}$-diethyl pyrocarbonate $\left(\mathrm{H}_{2} \mathrm{O}-\mathrm{DEPC}\right)$. The plates were 
Table 1. Primer sequences used for miRNAs and qRT-PCR in this study

\begin{tabular}{lll}
\hline ID & Sense primer $\left(5^{\prime} \rightarrow 3^{\prime}\right)$ & Antisense primer $\left(5^{\prime} \rightarrow 3^{\prime}\right)$ \\
\hline miR-375-3p & CGCCGTTTGTTCGTTCGGCT & CAGCCACAAAAGAGCACAAT \\
miR-375-3p stem loop primer & CCTGTTGTCTCCAGCCACAAAAGAGCACAATATTTCAGGAGACAACAGGTCACGCG & \\
U6 & CTCGCTTCGGCAGCACA & AACGCTTCACGAATTTGCGT \\
miR-375-3p mimic & UUUGUUCGUUCGGCUCGCGUGA & ACGCGAGCCGAACGAACAAAUU \\
Negative control & UUCUCCGAACGUGUCACGUTT & ACGUGACACGUUCGGAGAATT \\
miR-375-3p inhibitor & UCACGCGAGCCGAACGAACAAA & \\
miRNA inhibitor negative control & CAGUACUUUUGUGUAGUACAA &
\end{tabular}

incubated for $15 \mathrm{~min}$ at $16^{\circ} \mathrm{C}, 1 \mathrm{~h}$ at $37^{\circ} \mathrm{C}, 5 \mathrm{~min}$ at $85^{\circ} \mathrm{C}$, and finally maintained at $4{ }^{\circ} \mathrm{C}$. The qRT-PCR analysis of the miRNAs was performed with SYBR Green I dye according to the manufacturer's protocol. The mixture used for the amplification contained $1 \mu \mathrm{L}$ of complementary DNA (cDNA), $5 \mu \mathrm{L}$ of ChamQ SYBR qPCR master mix, and $0.2 \mu \mathrm{L}$ of $10 \mu \mathrm{M}$ primers (forward and reverse primers, mRNA; forward primers, miRNA) in a volume of $10 \mu \mathrm{L}$ via the addition of $\mathrm{H}_{2} \mathrm{O}$-DEPC. The CDNA samples were amplified in 96well optical reaction plates incubated in a CFX96 Real-Time PCR Detection System (Bio-Rad) for $30 \mathrm{~s}$ at $94^{\circ} \mathrm{C}$, followed by 40 cycles of $5 \mathrm{~s}$ at $94^{\circ} \mathrm{C}$ and $30 \mathrm{~s}$ at $60^{\circ} \mathrm{C}$. The fluorescence signal was generated by SYBR Green I DNA dye, and the intensity was measured during annealing steps. The specificity of the amplification was recorded and analyzed by a melting curve. The data were collected and recorded by the CFX Manager Software (Bio-Rad), and expressed as a function of the threshold cycle (Ct). The qRTPCR samples were evaluated using a single predominant peak as a quality control. The relative expression of the miRNAs was determined by the $\mathrm{Ct}\left(2^{-\Delta \Delta \mathrm{Ct}}\right)$ method.

Statistical analysis

All data are expressed as the mean \pm SEM. Statistical significance was determined by a one-way analysis of variance, followed by Dunnett's multiple comparison tests. In addition, a $t$ test was performed to analyze the differences in the data between two groups. $P<0.05$ was considered statistically significant. The figures were obtained by the GraphPad Software (GraphPad Prism 5, GraphPad Software, San Diego, CA, USA).

\section{RESULTS}

Magnesium isoglycyrrhizinate attenuates fructose-induced EMT and liver fibrosis

In fructose-fed rats, the serum levels of ALT (Fig. 1a) and AST (Fig. 1b) as well as the urine (Fig. 1c) and serum (Fig. 1d) hyaluronic acid levels were significantly increased with liver dysfunction. The H\&E, Masson's trichrome, and Sirius red staining showed liver histological changes, including inflammatory cell infiltration and perisinusoidal or portal/peripotal fibrosis, in the fructose-fed rats (Fig. 1e). The liver fibrotic area was significantly increased (Fig. 1f), and the liver fibrosis grade was aggravated in this animal model (Table 2).

Furthermore, the E-Cadherin (an epithelial marker) was significantly decreased, but the a-SMA, vimentin, N-Cadherin (as fibroblast marker), Collagen 1 , and fibronectin (fibrotic matrix components) were significantly increased at protein levels in the liver of the fructose-fed rats (Fig. 2a). The hepatic immunohistofluorescence and immunohistochemistry staining also showed the loss of E-Cadherin and an increase in $\mathrm{N}$-Cadherin and vimentin in this animal model (Fig. 2b, c).

To further confirm that fructose induced an EMT state in fibrosis, BRL-3A cells and primary rat hepatocytes were exposed to $5 \mathrm{mM}$ fructose. The E-Cadherin protein levels were decreased, while the a-SMA protein levels were remarkably increased in these cell models (Fig. 3a, c). To determine whether the fructose-exposed BRL-3A cells and primary rat hepatocytes possess mesenchymal function, the Collagen 1 and fibronectin protein levels were determined and found to be significantly increased (Fig. 3a, c). Consistently, the immunofluorescence staining showed that the E-Cadherin expression was reduced, while the a-SMA, vimentin, and Collagen 1 expression was increased in these cell models (Fig. 3b, d).

Magnesium isoglycyrrhizinate and pioglitazone could significantly prevent the excessive fructose-induced liver pathological damage in the rats (Fig. 1), displaying an anti-liver fibrosis effect with improvement in liver function. Moreover, they remarkably increased the protein levels of E-Cadherin and decrease the protein levels of a-SMA, vimentin, $\mathrm{N}$-Cadherin, Collagen 1 and fibronectin in the fructose-fed rat livers (Fig. 2) and fructoseexposed BRL-3A cells and primary rat hepatocytes (Fig. 3 ). These results indicate that magnesium isoglycyrrhizinate effectively ameliorates high fructose-induced EMT in liver fibrosis.

Magnesium isoglycyrrhizinate inhibits TGF- $\beta 1 /$ Smads signaling activation

Compared with the control group, the protein levels of TGF- $\beta 1, p-$ Smad2, p-Smad3, and Smad4 were significantly increased in the fructose-fed rat livers (Fig. 4a) and fructose-exposed BRL-3A cells (Fig. 4b) and primary rat hepatocytes (Fig. 4c). Magnesium isoglycyrrhizinate and pioglitazone reduced the fructose-induced upregulation of TGF- $\beta 1$, p-Smad2, p-Smad3, and Smad4 in these animal and cell models (Fig. 4), showing the suppression of TGF$\beta 1 /$ Smads signaling activation.

Magnesium isoglycyrrhizinate restrains the JAK2/STAT3 pathway to suppress TGF- $\beta 1 / \mathrm{Smad}$ signaling activation

In this study, a significant increase of hepatic JAK2, p-JAK2, and pSTAT3 protein levels was detected in the fructose-fed rats compared with those in the control group (Fig. 5a). Furthermore, the JAK2/STAT3 pathway was activated in the fructose-exposed BRL-3A cells (Fig. 5b) and primary rat hepatocytes (Fig. 5c). Subsequently, to explore the association between the JAK2/STAT3 pathway and TGF- $\beta 1 / \mathrm{Smad}$ signaling, the BRL-3A cells were treated with or without $5 \mathrm{mM}$ fructose in the presence or absence of $50 \mu \mathrm{M}$ JAK2 inhibitor AG490 for $6 \mathrm{~h}$. AG490 abolished the fructose-induced increase in p-JAK2, p-STAT3, TGF- $\beta 1$, p-Smad2, pSmad3, Smad4, a-SMA, and Collagen 1, and decrease in ECadherin in the BRL-3A cells (Fig. 6), indicating that the inhibition of the JAK2/STAT3 pathway represses TGF- $\beta 1 / \mathrm{Smad}$ signaling in the attenuation of the EMT.

Magnesium isoglycyrrhizinate and pioglitazone were found to down-regulate the JAK2, p-JAK2, and p-STAT3 protein levels in fructose-fed rat livers (Fig. $5 \mathrm{a}$ ) and fructose-exposed BRL-3A cells (Fig. 5b) and primary rat hepatocytes (Fig. 5c). Moreover, magnesium isoglycyrrhizinate was found to restrain the fructoseinduced upregulation of p-JAK2, p-STAT3, TGF- $\beta 1, p-S m a d 2, p-$ Smad3, Smad4, a-SMA, and Collagen 1, and downregulation of E-Cadherin in BRL-3A cells treated with AG490 (Fig. 6). 
a

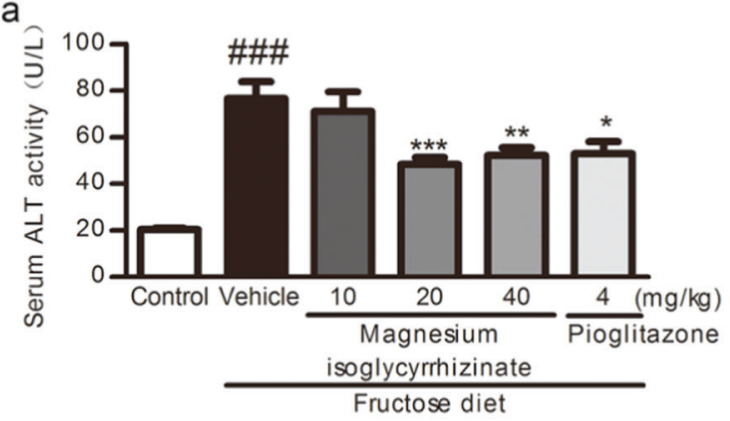

C

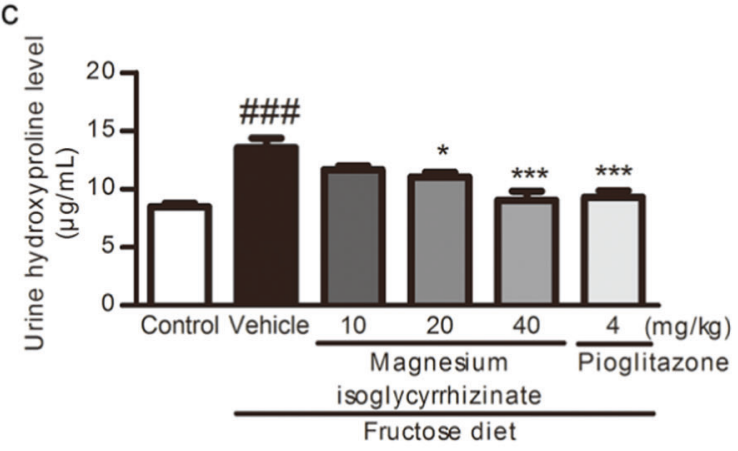

b

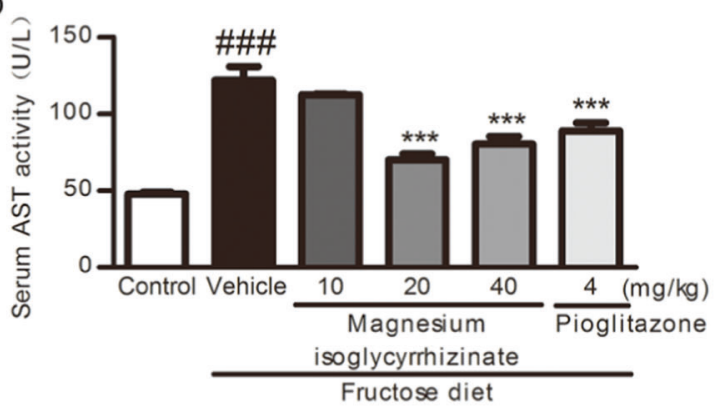

d

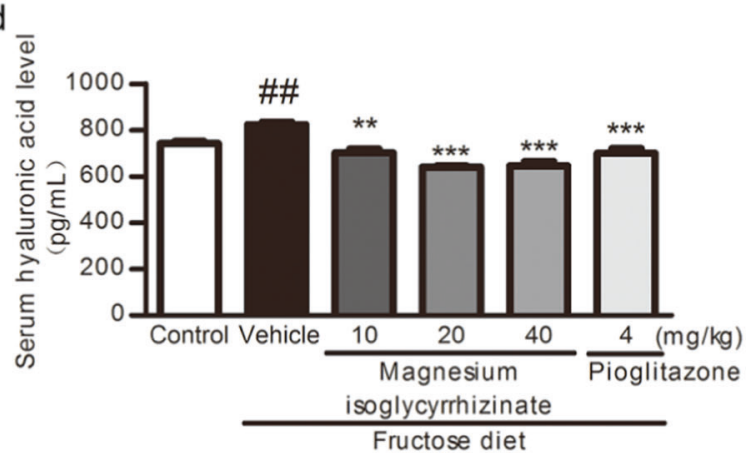

Fructose diet

e
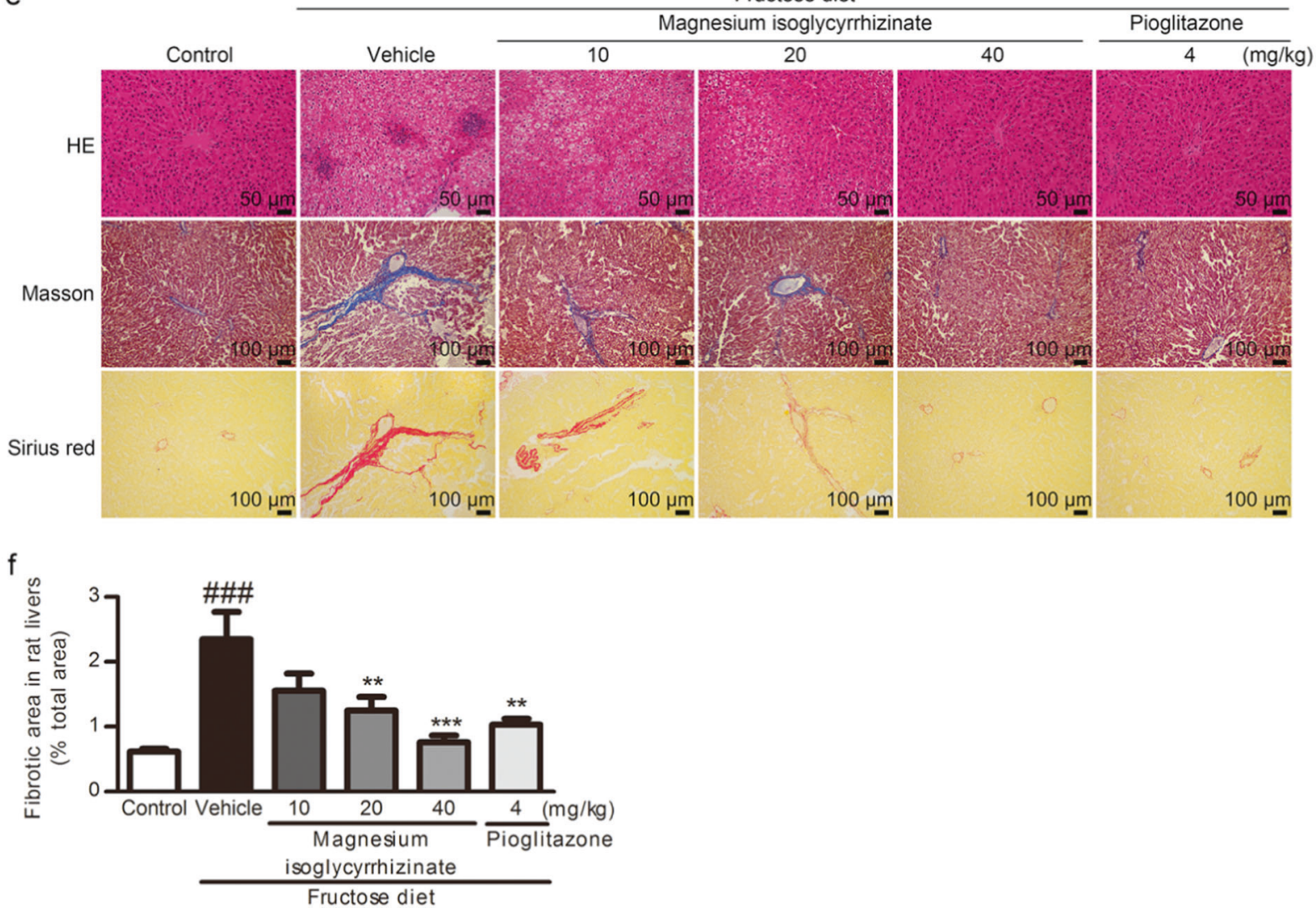

Fig. 1 Magnesium isoglycyrrhizinate alleviates liver fibrosis in high fructose-fed rats. Serum levels of ALT (a) and AST (b), serum (c) and urine (d) levels of hydroxyproline were measured at 17 weeks using standard diagnostic kits $(n=7)$. e Representative images of H\&E-stained (scale bar, $50 \mu \mathrm{m}$ ), Masson-stained (scale bar, $100 \mu \mathrm{m}$ ) and Sirius red-stained (scale bar, $100 \mu \mathrm{m}$ ) paraffin-embedded sections of liver tissues are shown. f Fibrotic areas in Sirius red-stained liver sections quantitatively compared $(n=6)$. The data are expressed as the mean \pm SEM. ${ }^{\# \#} P<$ $0.01,{ }^{\# \# \# P} P<0.001$ versus Control-Vehicle; ${ }^{*} P<0.05$, ${ }^{* *} P<0.01$, ${ }^{* *} P<0.001$ versus Fructose-Vehicle 
Table 2. Grades of rat liver fibrosis in each group according to the Ishak criterion

\begin{tabular}{lll}
\hline Group & Grade & Description \\
\hline Normal-Control & 0 & No fibrosis \\
$10 \%$ Fructose & 1 & Fibrous expansion of some portal areas, with short fibrous septa \\
Vehicle & 1 & Fibrous expansion of some portal areas, with short fibrous septa \\
Magnesium isoglycyrrhizinate $(10 \mathrm{mg} / \mathrm{kg})$ & 1 & Fibrous expansion of some portal areas, with short fibrous septa \\
Magnesium isoglycyrrhizinate $(20 \mathrm{mg} / \mathrm{kg})$ & 0 & No fibrosis \\
Magnesium isoglycyrrhizinate $(40 \mathrm{mg} / \mathrm{kg})$ & 0 & No fibrosis \\
Pioglitazone $(4 \mathrm{mg} / \mathrm{kg})$ & &
\end{tabular}

Pioglitazone had similar effects in these cell models (Fig. 6). These data indicate that magnesium isoglycyrrhizinate may attenuate high fructose-induced EMT in liver fibrosis at least partially by inhibiting the JAK2/STAT3 pathway to restrain TGF- $\beta 1 / \mathrm{Smad}$ signaling activation.

Magnesium isoglycyrrhizinate increases miR-375-3p expression to partially prevent the JAK2/STAT3 pathway and TGF- $\beta 1 / \mathrm{Smads}$ signaling activation

Low miR-375-3p expression was detected in fructose-fed rat livers (Fig. 7a) and fructose-exposed BRL-3A cells (Fig. 7b) and primary rat hepatocytes (Fig. 7c). Searching the TargetScan database revealed putative miR-375-3p binding sites on the $3^{\prime}$-UTR of JAK2 mRNA (Fig. $7 \mathrm{~g}$ ). The JAK2 mRNA levels were also increased in these animal and cell models (Fig. 7d-f). To explore the relationship between miR-375-3p and JAK2, BRL-3A cells were transfected with a miR-375-3p mimic or miR-375-3p inhibitor for $6 \mathrm{~h}$. The transfection efficiency of miR-375-3p mimic (Fig. 8a) or miR-375-3p inhibitor (Fig. 9a) was assayed by qRT-PCR. The luciferase activity in the BRL-3A cells transfected with the miRGLOJAK2-3'-UTR WT and miR-375-3p mimic was significantly decreased compared with that in the cells transfected with the miRGLO-JAK2-3'-UTR WT and miR-375-3p normal control, while the BRL-3A cells transfected with the miRGLO-JAK2-3'-UTR mutant type and miR-375-3p mimic did not show a significant difference (Fig. 7h). Of note, the JAK2 protein and mRNA levels were greatly decreased by the miR-375-3p mimic transfection (Fig. 8c, e), while the miR-375-3p inhibitor-transfected BRL-3A cells showed a significant upregulation of the JAK2 protein and mRNA levels (Fig. 9c, e). Therefore, low miR-375-3p expression may induce JAK2 upregulation in hepatocytes.

To further investigate the role of miR-375-3p in the JAK2/ STAT3 pathway and TGF- $\beta 1 / \mathrm{Smads}$ signaling, BRL-3A cells were transfected with a miR-375-3p mimic or inhibitor for $6 \mathrm{~h}$, and then incubated with or without fructose for another 24 and $48 \mathrm{~h}$. The transfection with the miR-375-3p mimic significantly increased miR-375-3p expression in the BRL-3A cells under high fructose condition (Fig. 8d). The high fructose-induced increase in JAK2, p-JAK2, p-STAT3, TGF- $\beta 1$, p-Smad2, p-Smad3, Smad4, aSMA, and Collagen 1, and decrease in E-Cadherin in the BRL-3A cells were attenuated by the miR-375-3p mimic (Fig. 8e-g). These observations indicate that high miR-375-3p expression may inhibit the activation of the JAK2/STAT3 pathway and downstream TGF- $\beta 1 / \mathrm{Smad}$ signaling, possibly resulting in the attenuation of high fructose-induced EMT. However, the miR$375-3 p$ inhibitor reduced miR-375-3p expression under the high fructose condition (Fig. 9d). The miR-375-3p inhibitor also increased JAK2, p-JAK2, p-STAT3, TGF- 1 1, p-Smad2, p-Smad3, Smad4, a-SMA, and Collagen 1 and decreased the E-Cadherin protein levels in the BRL-3A cells exposed to high fructose (Fig. 9e-g), indicating that low miR-375-3p expression may activate the JAK2/STAT3 pathway to trigger downstream TGF-B1/ Smad signaling, causing the EMT.
Magnesium isoglycyrrhizinate and pioglitazone upregulated miR-375-3p expression in fructose-fed rat livers, fructose-exposed BRL-3A cells, and primary rat hepatocytes (Fig. $7 a-c)$. In the miR375-3p mimic-transfected BRL-3A cells, the high fructose-induced low expression of miR-375-3p (Fig. 8e), upregulation of JAK2, p-JAK2, p-STAT3, TGF- 31 , p-Smad2, p-Smad3, Smad4, a-SMA, and Collagen 1, and downregulation of the E-Cadherin were attenuated (Fig. $8 \mathrm{e}-\mathrm{g}$ ). In the BRL-3A cells transfected with the miR-375$3 p$ inhibitor, magnesium isoglycyrrhizinate and pioglitazone could increase miR-375-3p expression (Fig. 9d), and restored miR-375$3 p$ inhibitor-induced an increase in JAK2, p-JAK2, p-STAT3, TGF- $\beta 1$, p-Smad2, p-Smad3, Smad4, a-SMA, and Collagen 1, and decrease in the E-Cadherin under high fructose condition (Fig. 9e-g). Thus, magnesium isoglycyrrhizinate may ameliorate high fructose-induced EMT in liver fibrosis by upregulating miR375-3p expression to reduce JAK2/STAT3 pathway-activated TGF$\beta 1 /$ Smad signaling (Fig. 10).

\section{DISCUSSION}

Fibrosis is associated with the progression of chronic liver disease. However, no specific therapy is available to recover liver fibrosis. Magnesium isoglycyrrhizinate is a hepatoprotective agent that improves liver function and treats chronic liver disease with few side effects [38]. Magnesium isoglycyrrhizinate also reduces the hyaluronic acid and TGF- $\beta 1$ levels and prevents hepatic fibrosis in patients with chronic type B hepatitis [27]. However, the mechanisms by which magnesium isoglycyrrhizinate alleviates liver fibrosis remain unclear. Here, we showed that magnesium isoglycyrrhizinate significantly attenuated high fructose-induced EMT and liver fibrosis in rats, which is partially consistent with another report investigating carbon tetrachloride-induced liver fibrosis in rats [28]. Furthermore, magnesium isoglycyrrhizinate was confirmed to increase miR-375-3p expression to inhibit the JAK2/STAT3 pathway and TGF- $\beta 1 /$ Smad signaling activation, resulting in a reduction in the EMT in high fructose-exposed BRL-3A cells and primary rat hepatocytes. Our findings indicate that magnesium isoglycyrrhizinate may interact with miR-375-3p to suppress the JAK2/STAT3 pathway and TGF- $\beta 1 /$ Smad signaling activation, attenuating high fructose-driven liver fibrosis.

Clinical trials and animal experiments have shown the close connection between excessive fructose intake and liver fibrosis [39-41]. In this study, liver fibrosis was observed in high fructosefed rats. The E-Cadherin protein levels were decreased, while the aSMA, Collagen 1, and fibronectin protein levels were increased in the rat livers, BRL-3A cells, and primary rat hepatocytes under the high fructose conditions, indicating a partial EMT in high fructoseinduced liver fibrosis. Magnesium isoglycyrrhizinate was found to reverse these changes in these animal and cell models. TGF- $\beta 1$ is reported to be a potent inducer of the EMT, primarily via a canonical Smad-dependent mechanism, to cause liver profibrogenic pathway and fibrosis [42]. Generally, via a rapid response to liver injury, TGF- $\beta 1$ synthesis and secretion occur, predominantly in 
a

Normal-Control

Fructose-Vehicle

Fructose diet + Magnesium isoglycyrrhizinate $(10 \mathrm{mg} / \mathrm{kg})$

Fructose diet + Magnesium isoglycyrrhizinate $(20 \mathrm{mg} / \mathrm{kg})$

Fructose diet + Magnesium isoglycyrrhizinate $(40 \mathrm{mg} / \mathrm{kg})$ Fructose diet + Pioglitazone $(4 \mathrm{mg} / \mathrm{kg})$

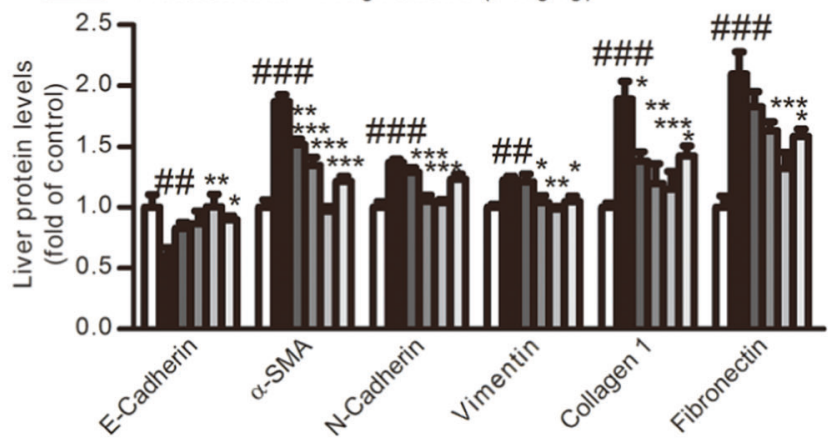

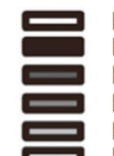

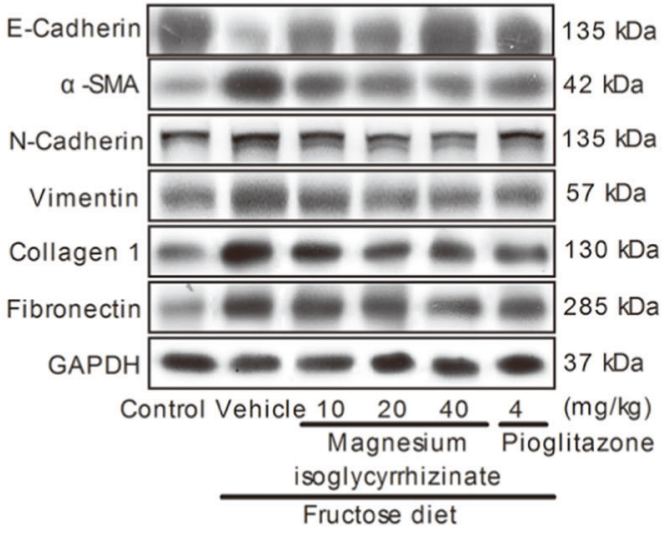

Fructose diet

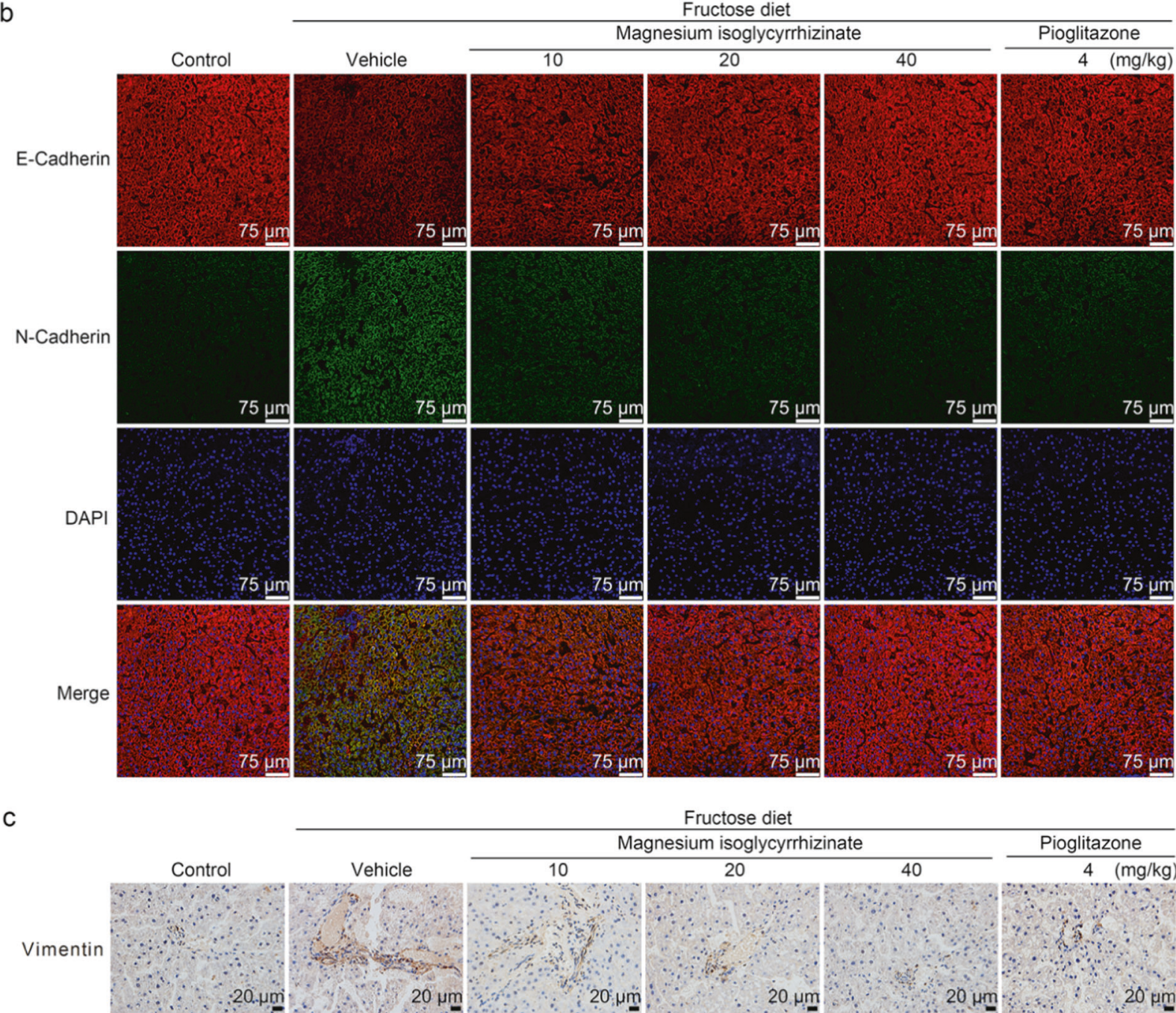

Fig. 2 Magnesium isoglycyrrhizinate ameliorates the EMT in high fructose-fed rat livers. a Protein levels of E-Cadherin, $\alpha$-SMA, N-Cadherin, vimentin, Collagen 1 , and fibronectin in rat livers were detected and measured by a Western blot analysis and normalized to GAPDH ( $n=6$ ). b Immunohistofluorescence analysis of E-Cadherin (red) and N-Cadherin (green) in rat liver sections. Representative images are shown (scale bar, $75 \mu \mathrm{m})$. c Immunohistochemistry analysis of vimentin in rat liver sections. Representative images are shown (scale bar, $20 \mu \mathrm{m})$. The data are expressed as the mean \pm SEM $(n=6)$. ${ }^{\# \#} P<0.01,{ }^{\# \# \#} P<0.001$ versus Normal-Control; ${ }^{*} P<0.05,{ }^{* *} P<0.01,{ }^{* * *} P<0.001$ versus FructoseVehicle 


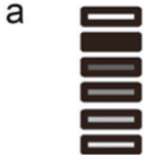

Normal-Control Fructose-Vehicle

Fructose $(5 \mathrm{mM})+$ Magnesium isoglycyrrhizinate $(10 \mu \mathrm{M})$

Fructose $(5 \mathrm{mM})+$ Magnesium isoglycyrrhizinate $(20 \mu \mathrm{M})$

Fructose $(5 \mathrm{mM})+$ Magnesium isoglycyrrhizinate $(40 \mu \mathrm{M})$

Fructose $(5 \mathrm{mM})+$ Pioglitazone $(10 \mu \mathrm{M})$
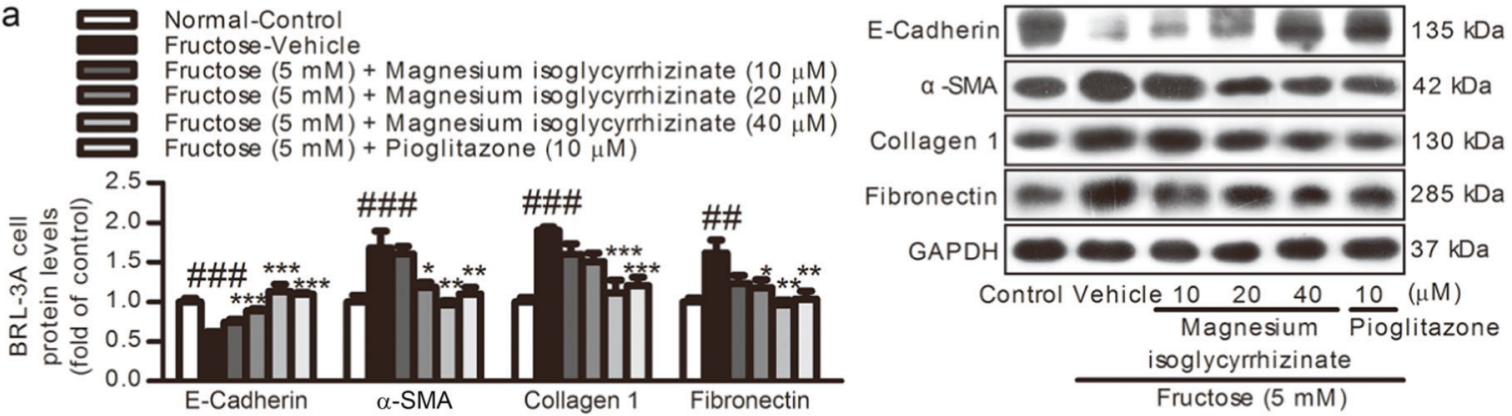

b

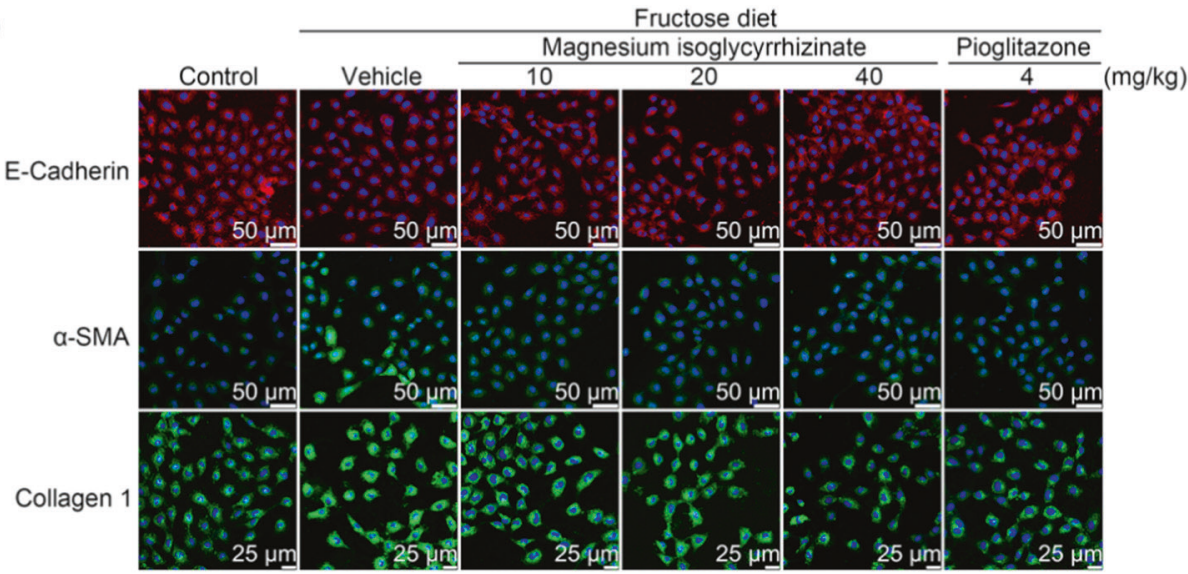

C
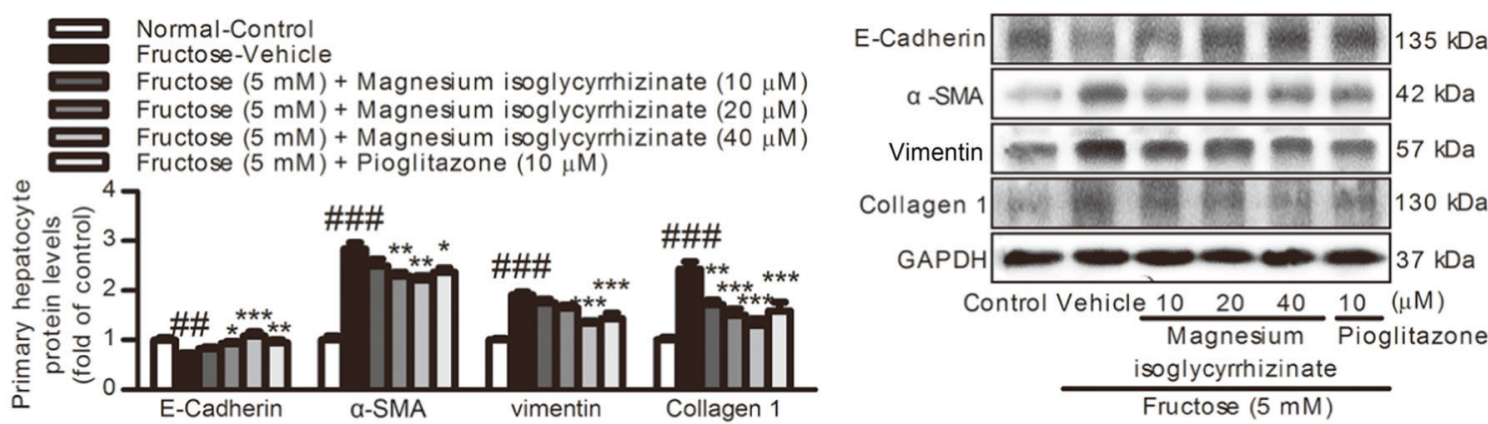

d

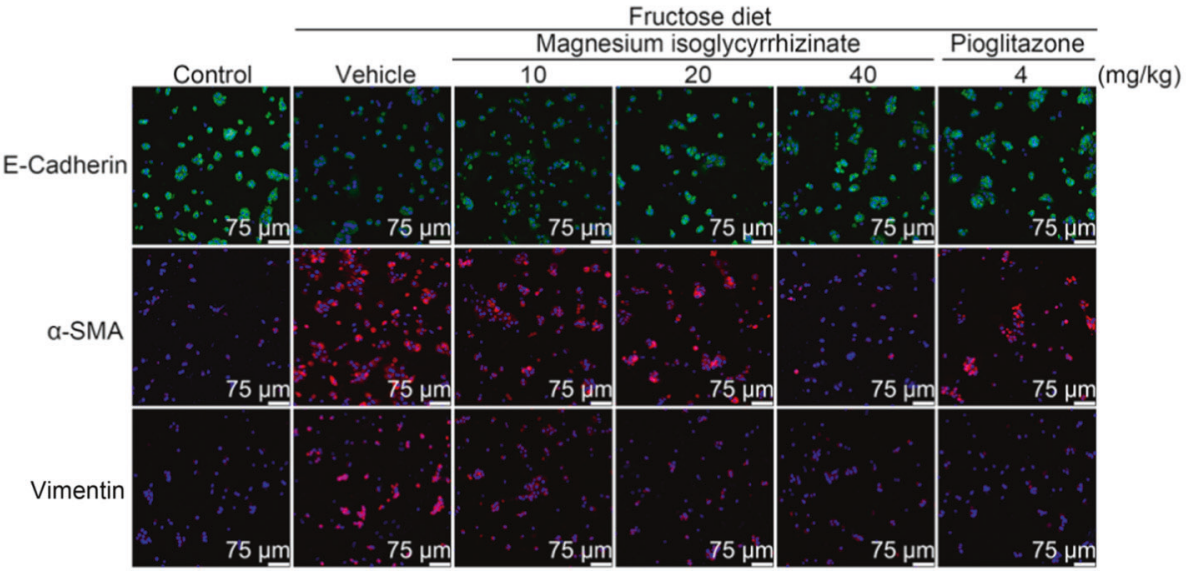

Fig. 3 Magnesium isoglycyrrhizinate attenuates high fructose-induced EMT in BRL-3A cells and primary rat hepatocytes. Protein levels of ECadherin, $\alpha$-SMA, Collagen 1, vimentin, and fibronectin in cell lysates of BRL-3A cells (a) and primary rat hepatocytes (c) were determined by a Western blot analysis. Relative protein levels were normalized to GAPDH $(n=6)$. Immunohistofluorescence analysis of E-Cadherin, $\alpha$-SMA, Collagen 1, and vimentin in BRL-3A cells $(\mathbf{b})$ and primary rat hepatocytes (d). Representative immunofluorescence images are shown (scale bar, $50 \mu \mathrm{m}$ for BRL-3A cells, $75 \mu \mathrm{m}$ for primary rat hepatocytes). The data are expressed as the mean \pm SEM $(n=6)$. ${ }^{\# \#} P<0.01$, ${ }^{\# \# \#} P<0.001$ versus Normal-Control; ${ }^{*} P<0.05,{ }^{* *} P<0.01,{ }^{* * *} P<0.001$ versus Fructose-Vehicle 
a

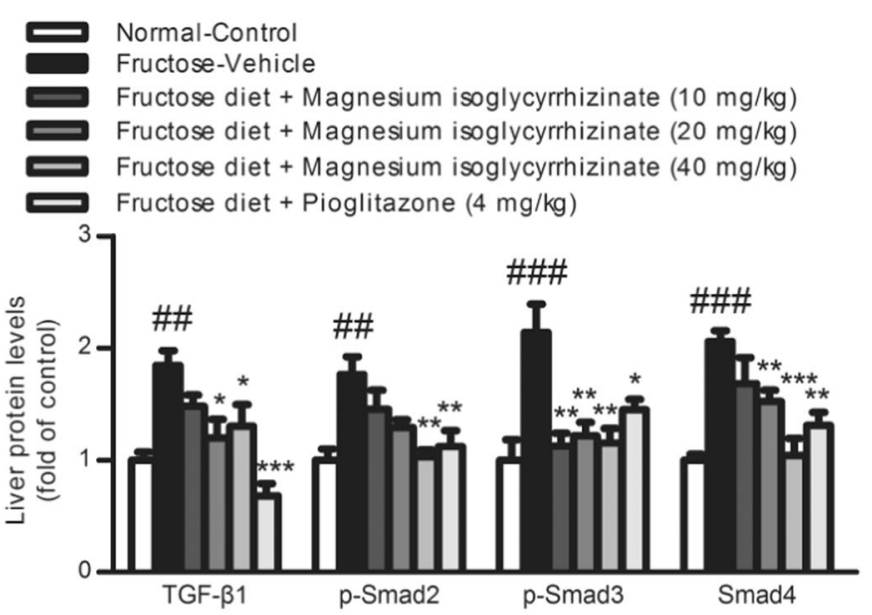

b

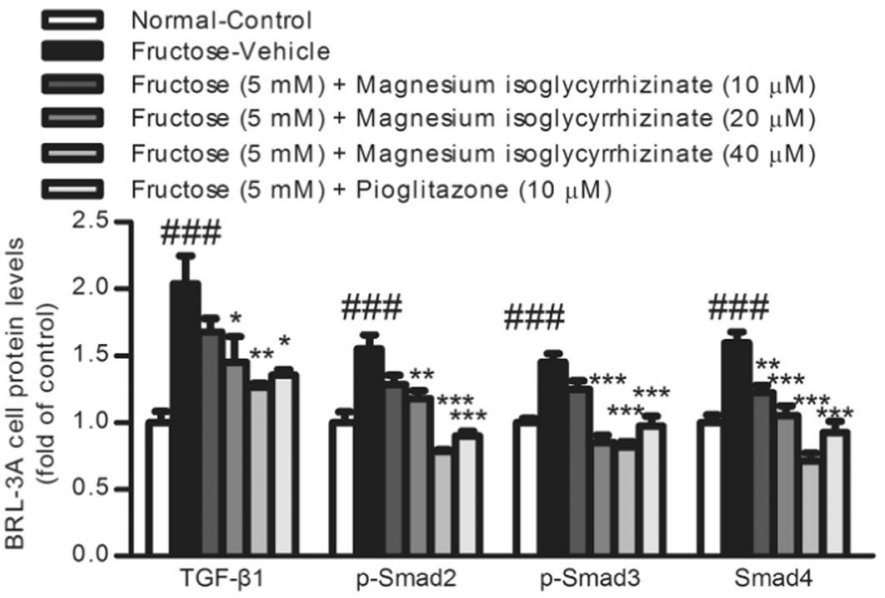

C

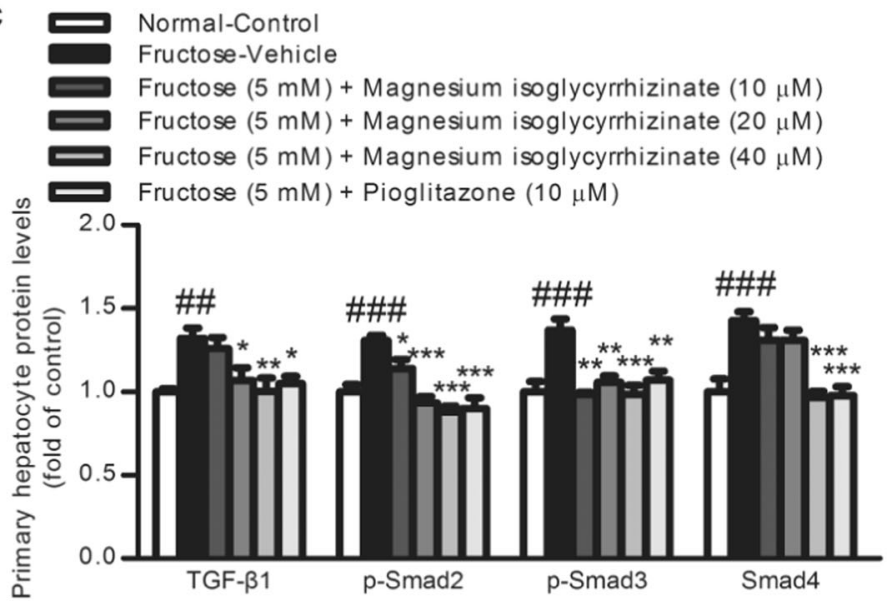

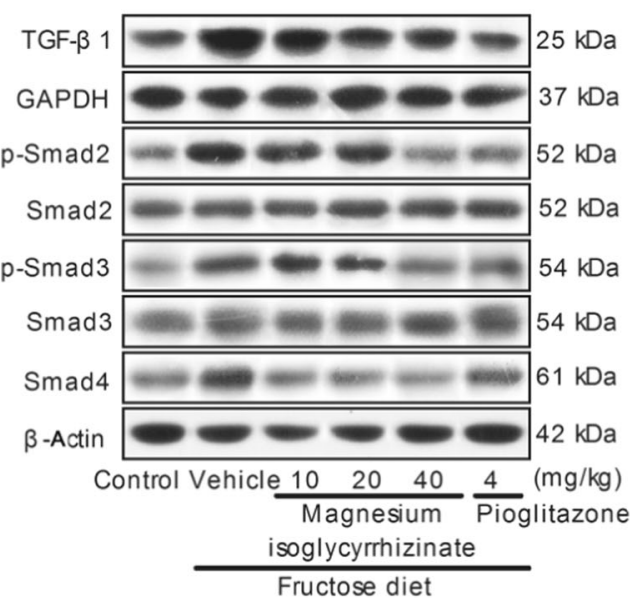
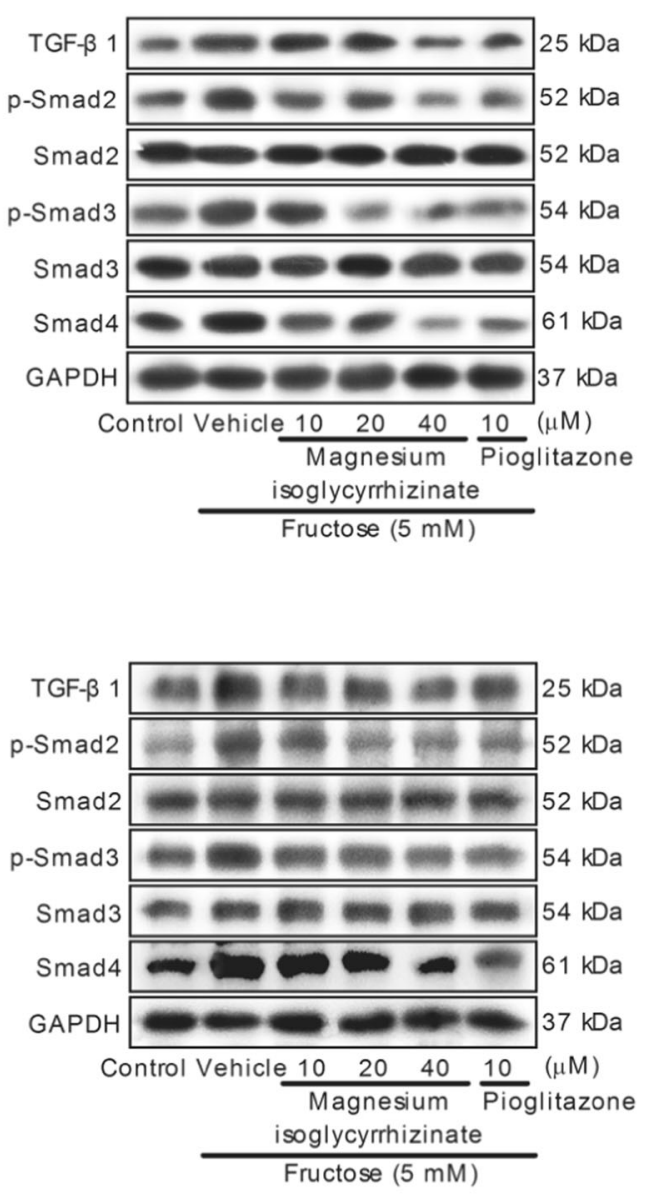

Fig. 4 Magnesium isoglycyrrhizinate inhibits TGF- $\beta 1 /$ Smad signaling activation in fructose-fed rat livers and fructose-exposed BRL-3A cells and primary rat hepatocytes. TGF- $\beta 1, \mathrm{p}-\mathrm{Smad} 2, \mathrm{p}-\mathrm{Smad} 3$, and Smad4 protein levels in rat livers (a) and cell lysates of BRL-3A cells (b) and primary rat hepatocytes (c) were determined by a Western blot analysis. Relative protein levels of TGF- $\beta 1$ and Smad4 were normalized to GAPDH and $\beta$-actin, and those of $\mathrm{p}$-Smad2 and $\mathrm{p}$-Smad3 were normalized to Smad 2 and Smad3, respectively. The data are expressed as the

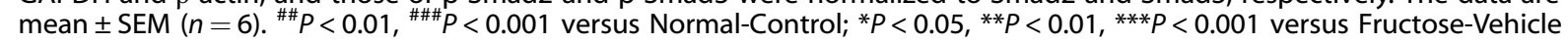

hepatic stellate cells, subsequently, TGF- $\beta 1$ induces quiescent mature hepatocytes to undergo the EMT [43-46]. Some of studies have emphasized the activation of hepatic stellate cells in fibrogenesis [47, 48]. However, in vivo evidence of hepatocyte EMT in carbon tetrachloride-induced liver fibrosis remains controversial; [49] it could be hypothesized that magnesium 
a
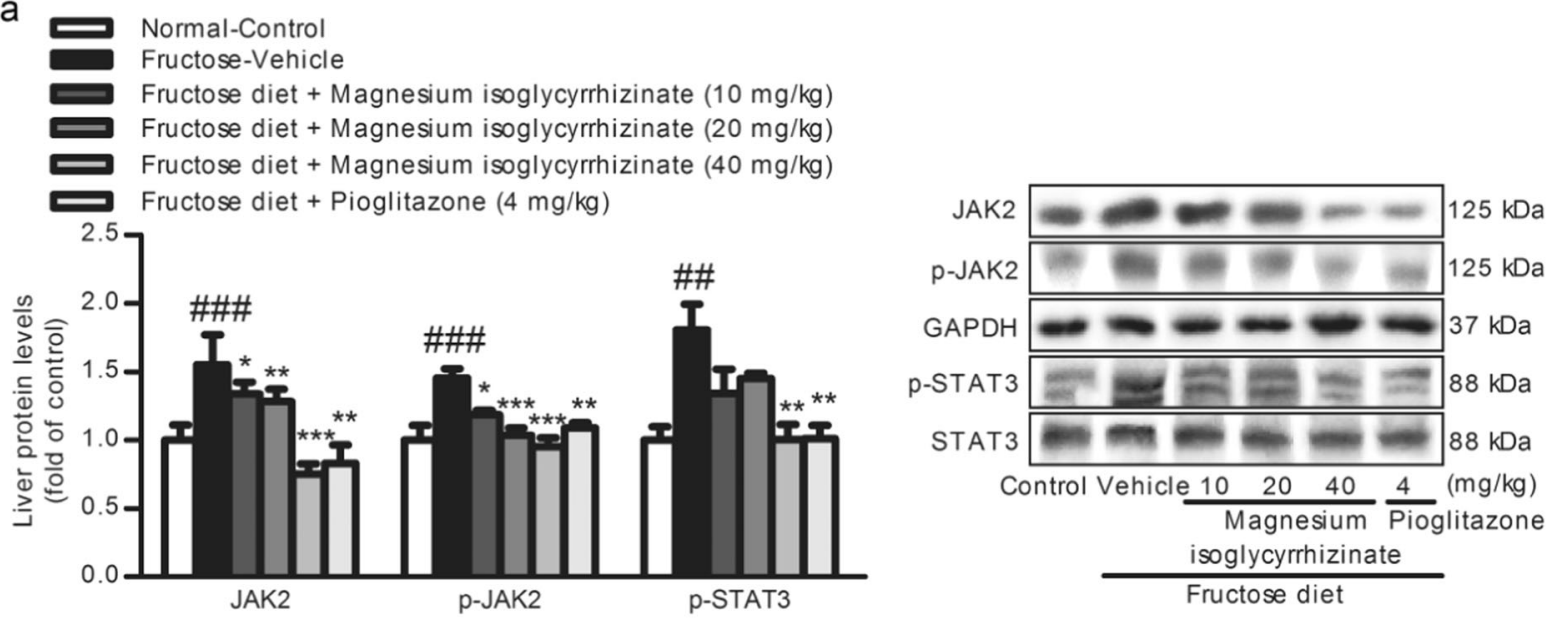
b Normal-Control
Fructose-Vehicle
Fructose $(5 \mathrm{mM})+$ Magnesium isoglycyrrhizinate $(10 \mu \mathrm{M})$
Fructose $(5 \mathrm{mM})+$ Magnesium isoglycyrrhizinate $(20 \mu \mathrm{M})$
Fructose $(5 \mathrm{mM})+$ Magnesium isoglycyrrhizinate $(40 \mu \mathrm{M})$
Fructose $(5 \mathrm{mM})+$ Pioglitazone $(10 \mu \mathrm{M})$
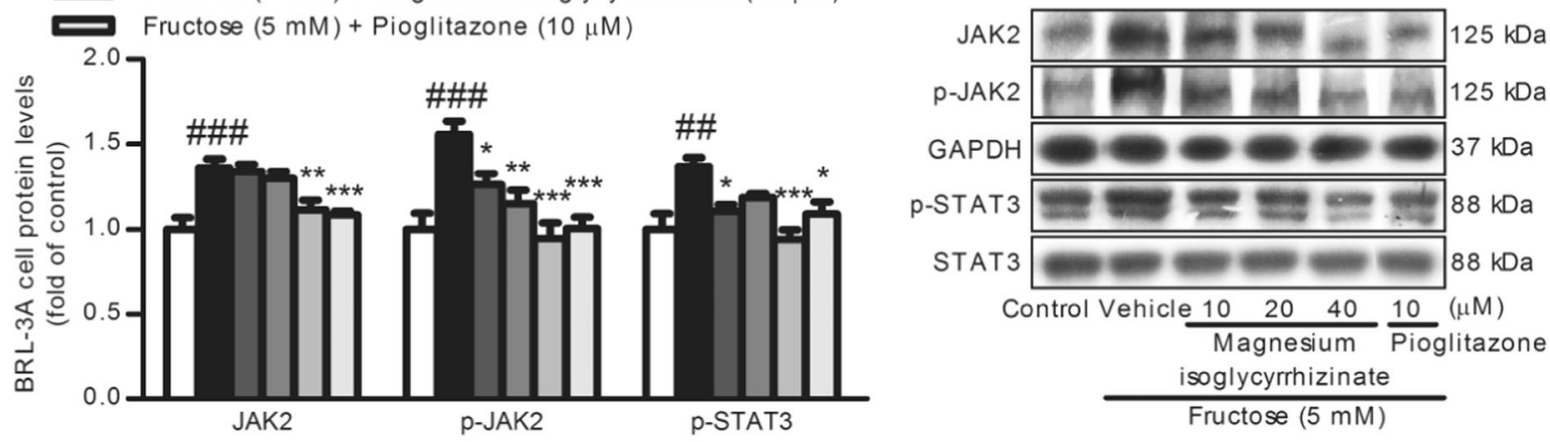
C Normal-Control
Fructose-Vehicle
Fructose $(5 \mathrm{mM})+$ Magnesium isoglycyrrhizinate $(10 \mu \mathrm{M})$
Fructose $(5 \mathrm{mM})+$ Magnesium isoglycyrrhizinate $(20 \mu \mathrm{M})$
Fructose $(5 \mathrm{mM})+$ Magnesium isoglycyrrhizinate $(40 \mu \mathrm{M})$
Fructose $(5 \mathrm{mM})+$ Pioglitazone $(10 \mu \mathrm{M})$
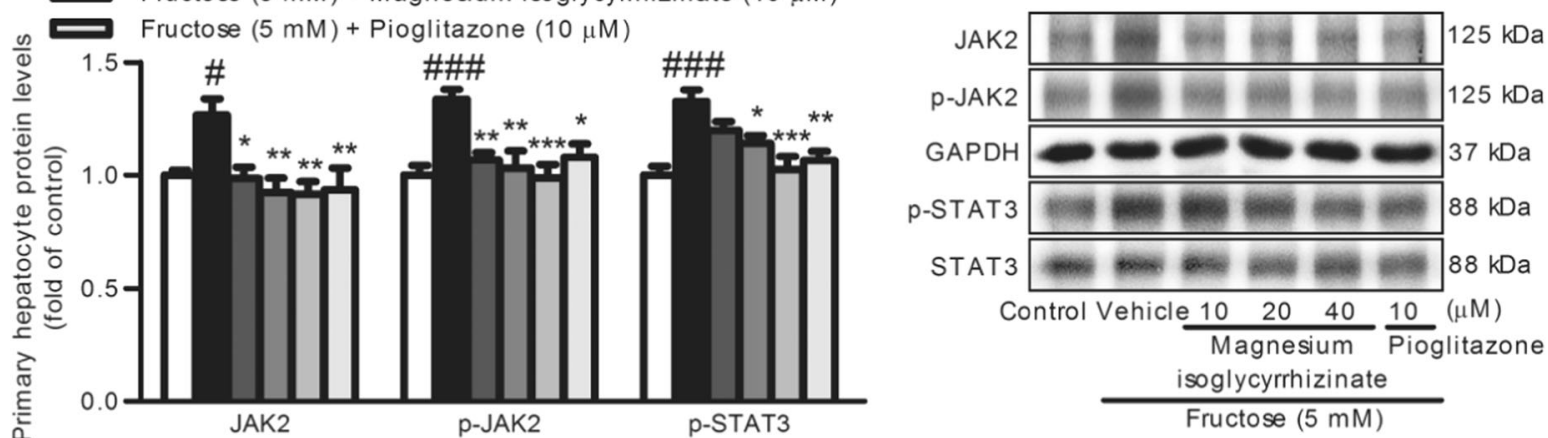

Fig. 5 Magnesium isoglycyrrhizinate suppresses the JAK2/STAT3 pathway in fructose-fed rat livers and fructose-exposed BRL-3A cells and primary rat hepatocytes. JAK2, p-JAK2, and p-STAT3 protein levels in rat livers (a) and cell lysates of BRL-3A cells (b) and primary rat hepatocytes (c) were determined by a Western blot analysis. Relative protein levels of JAK2 and p-JAK2 were normalized to GAPDH, and those of $p$-STAT3 were normalized to STAT3. The data are expressed as the mean \pm SEM $(n=6)$. ${ }^{\prime} P<0.05,{ }^{\# \#} P<0.01$, \#\#\# $P<0.001$ versus NormalControl; ${ }^{*} P<0.05,{ }^{* *} P<0.01,{ }^{* * *} P<0.001$ versus Fructose-Vehicle

Of note, JAK2 expression is increased in liver fibrosis of rodents and patients [4]. Interestingly, TGF- $\beta 1$ can inhibit interlukin- 6 signal transduction by decreasing the STAT3 phosphorylation levels in benign prostatic hyperplasia cells, which is associated with downregulation of the JAK2 mRNA and protein levels [50]. The inhibition of the JAK2/STAT3 pathway may decrease TGF- $\beta 1$ synthesis in experimental hepatic fibrosis [43]. Hepatic JAK2/ STAT3 pathway activation is observed in high fructose-fed rats [9]. Consistently, in this study, the activation of the JAK2/STAT3 pathway and TGF- $\beta 1 /$ Smads signaling was also detected in fructose-fed rat livers and fructose-exposed BRL-3A cells and primary rat hepatocytes. Furthermore, the JAK2 inhibitor AG490 

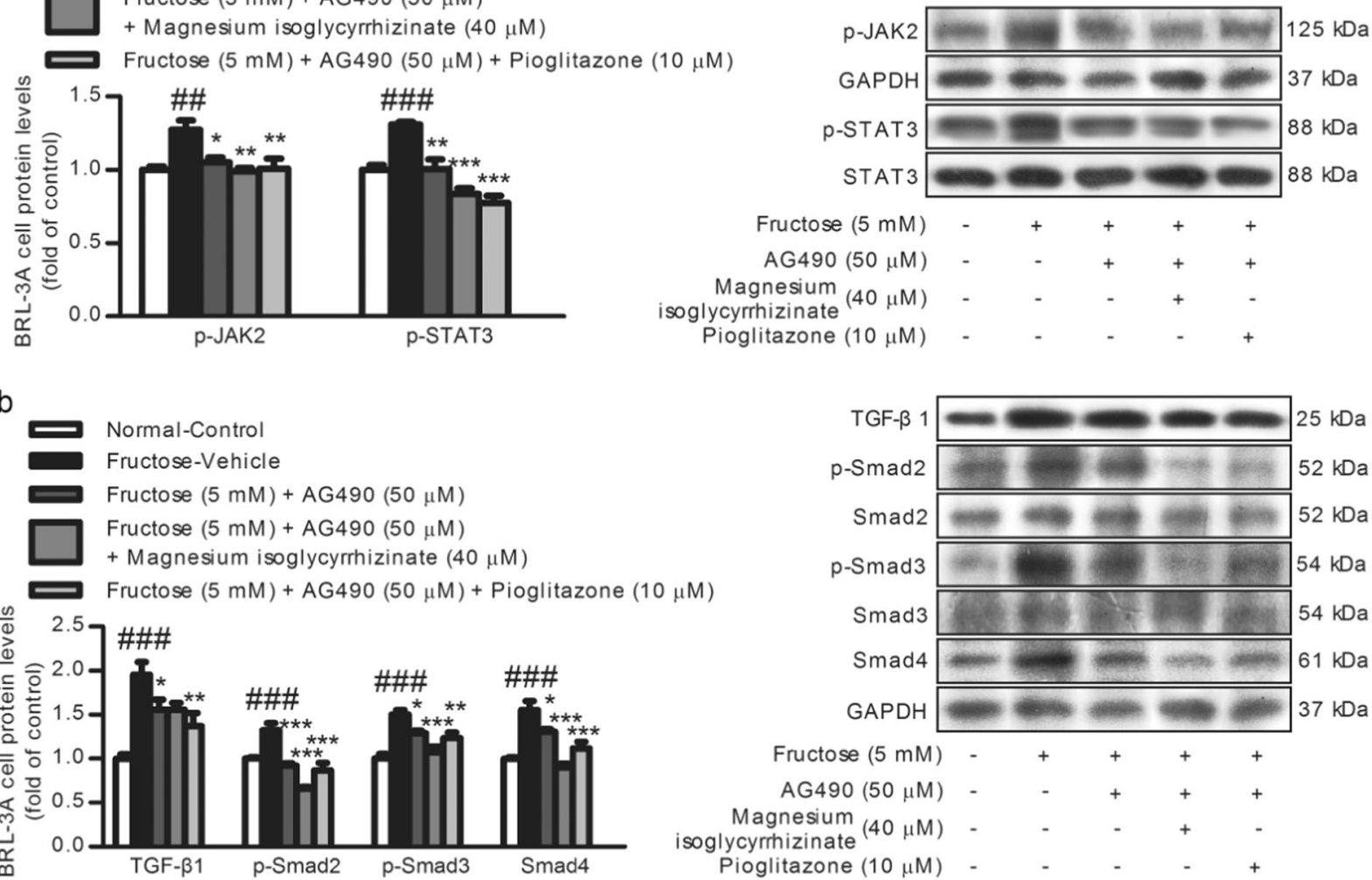

Fructose $(5 \mathrm{mM}) \quad-\quad+\quad+\quad+\quad+$

AG490 $(50 \mu \mathrm{M}) \quad-\quad-\quad+\quad+\quad+$ $\underset{\text { isoglycyrrhizinate }}{\text { Magnesium }}(40 \mu \mathrm{M}) \quad-\quad-\quad+\quad+$

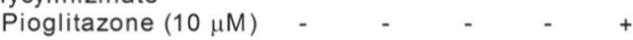

b TGF- $\beta$

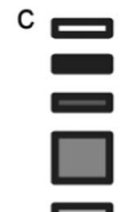

Normal-Control

Fructose-Vehicle

Fructose $(5 \mathrm{mM})+\mathrm{AG} 490(50 \mu \mathrm{M})$

Fructose $(5 \mathrm{mM})+\mathrm{AG} 490(50 \mu \mathrm{M})$

+ Magnesium isoglycyrrhizinate $(40 \mu \mathrm{M})$

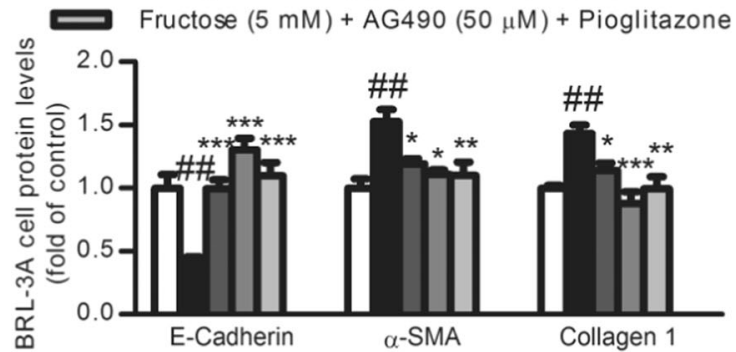

$(10 \mu \mathrm{M})$

$\alpha-$ SMA

Collagen 1

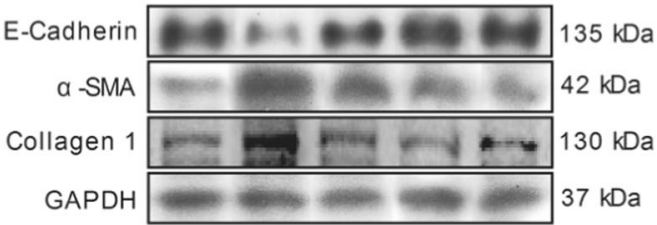

Fig. 6 Magnesium isoglycyrrhizinate inhibits the JAK2/STAT3 pathway to restrain TGF- $\beta 1 /$ Smads signaling in the EMT in high fructose-exposed BRL-3A cells. Protein levels of p-JAK2, p-STAT3 (a), TGF- $\beta 1, p-S m a d 2, p-S m a d 3$, Smad4 (b), and E-Cadherin, $\alpha$-SMA, and Collagen 1 (c) in cell lysates were determined by a Western blot analysis. Relative protein levels of p-JAK2, TGF- $\beta 1$, Smad4, E-Cadherin, $\alpha$-SMA, and Collagen 1 were normalized to GAPDH, and those of p-STAT3, p-Smad2, and p-Smad3 were normalized to STAT3, Smad2, and Smad3, respectively. The data are expressed as the mean \pm SEM $(n=6) .{ }^{\# \#} P<0.01,{ }^{\# \# \#} P<0.001$ versus Normal-Control; ${ }^{*} P<0.05,{ }^{* *} P<0.01,{ }^{* * *} P<0.001$ versus Fructose-Vehicle

abolished the fructose-induced change in TGF- $\beta 1 /$ Smads signaling in the BRL-3A cells, suggesting that the JAK2/STAT3 pathway may promote TGF- $\beta 1 /$ Smads signaling activation in high fructoseinduced fibrotic progression in hepatocytes. The impact of the direct interaction between the JAK2/STAT3 pathway and TGF- $\beta 1 /$ Smads signaling impacts on liver fibrosis in vivo warrants further investigation.

Interestingly, an abundant number of miRNAs have been reported to function as pro-fibrotic or antifibrotic agents in liver fibrosis [10]. Recently, low miR-375-3p expression was observed in liver biopsy tissues from class III obese human subjects with the progression of human nonalcoholic fatty liver disease [14]. Notably, putative miR-375-3p binding sites have been identified on the $3^{\prime}$-UTR of JAK2 mRNA in both humans and rats, prompting us to investigate the relationship between miR-375-3p and liver fibrosis following high fructose induction. Here, we found that miR-375-3p mimic reduced the high fructose-induced activation of the JAK2/STAT3 pathway, TGF- $\beta 1 /$ Smad signaling, and the EMT, while the miR-375-3p inhibitor promoted these high fructoseinduced changes in BRL-3A cells. Thus, low miR-375-3p 

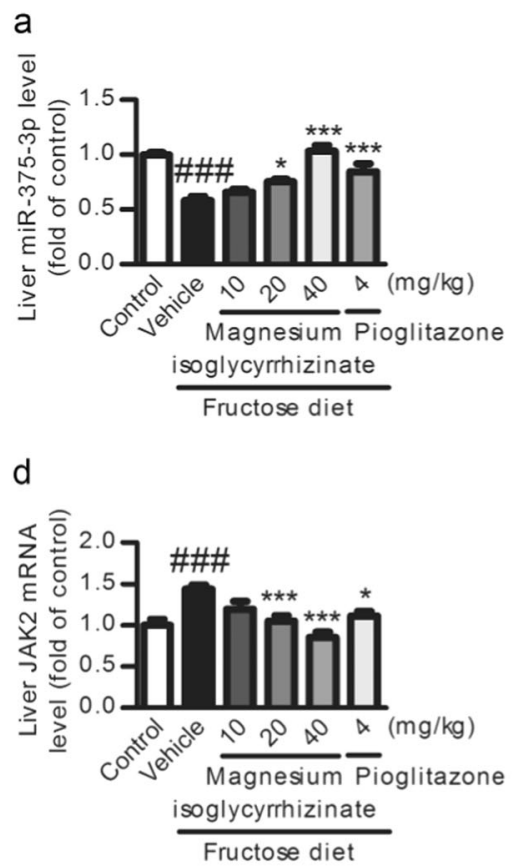
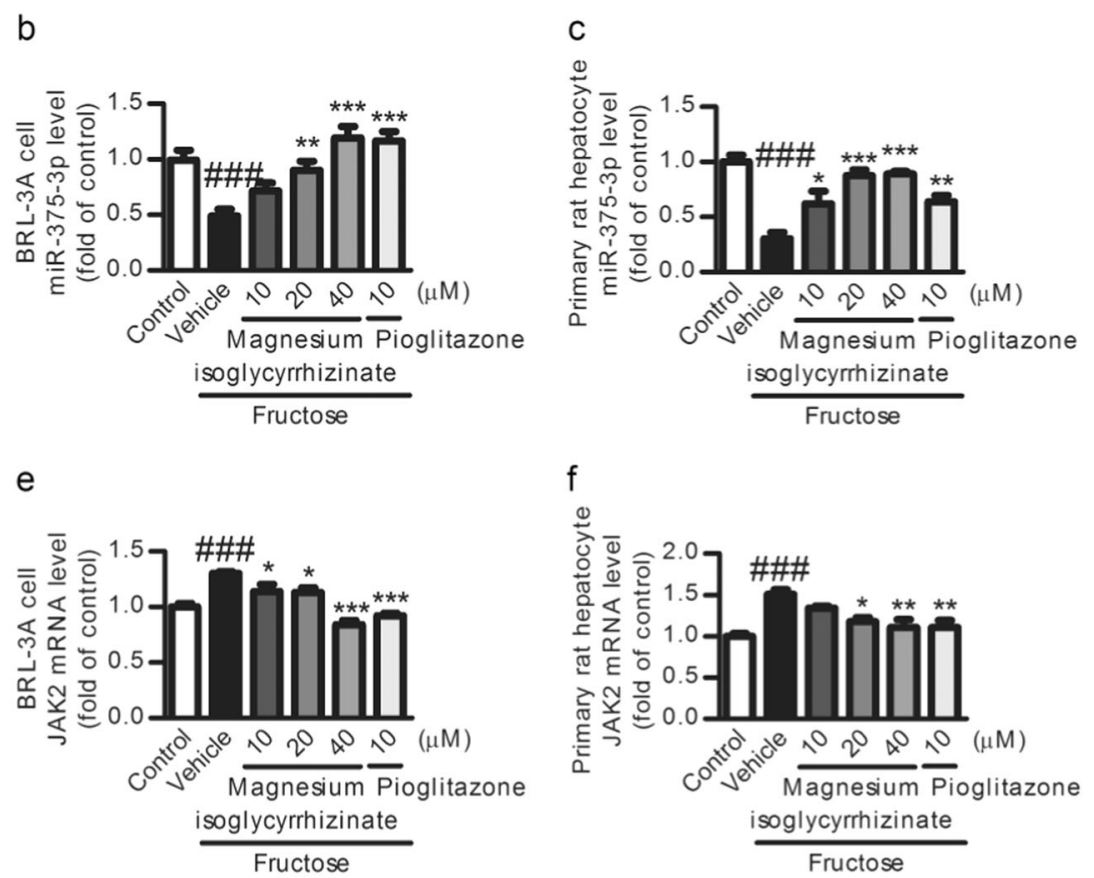

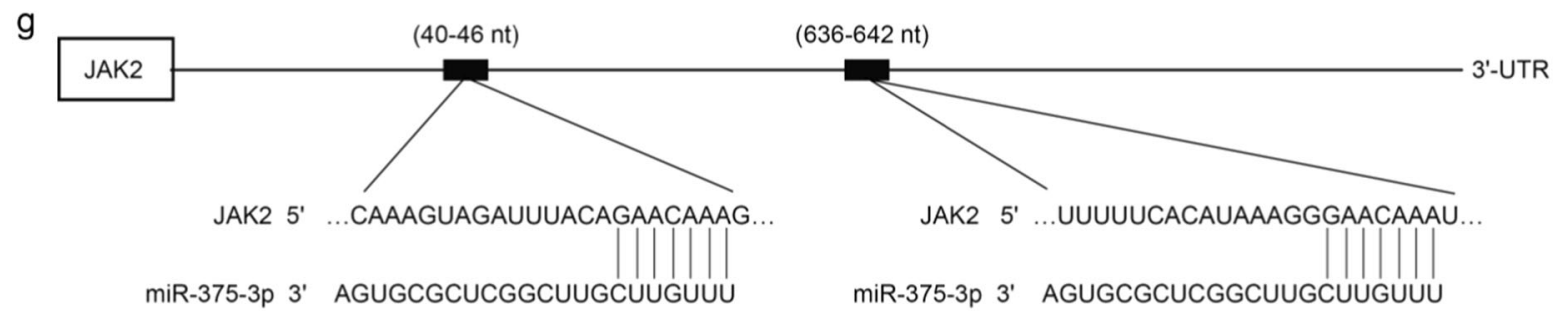

h

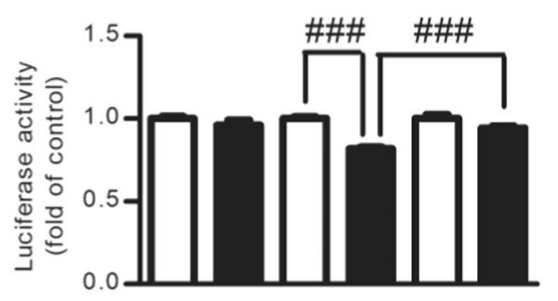

miRGLO $(1 \mu \mathrm{g} / \mathrm{mL})$

miRGLO-JAK2-3' UTR wt $(1 \mu \mathrm{g} / \mathrm{mL})$

miRGLO-JAK2-3' UTR mut (1 $\mu \mathrm{g} / \mathrm{mL})$

miR-375-3p negative control $(50 \mathrm{nM})$

miR-375-3p Mimic (50 nM)

Fig. 7 Magnesium isoglycyrrhizinate upregulates miR-375-3p to suppress the JAK2 mRNA level in fructose-fed rat livers and fructose-exposed BRL-3A cells and primary rat hepatocytes. MiR-375-3p levels in rat livers (a) and cell lysates of BRL-3A cells (b) and primary rat hepatocytes (c) were evaluated by a qRT-PCR analysis and normalized to U6 expression $(n=6)$. JAK2 mRNA levels in rat livers (d) and cell lysates of BRL-3A cells (e) and primary rat hepatocytes (f) were evaluated by a qRT-PCR analysis and normalized to GAPDH expression $(n=6)$. $\mathbf{g}$ Schematic of two putative miR-375-3p binding sites in the JAK2 3'-UTR region detected by TargetScan. $\mathbf{h}$ Luciferase reporter assay in BRL-3A cells. Relative firefly luciferase activity was normalized to Renilla luciferase activity. ${ }^{\# \#} P<0.001$ versus Normal-Control; ${ }^{*} P<0.05,{ }^{* *} P<0.01,{ }^{* * *} P<0.001$ versus Fructose-Vehicle

expression may provide a new pathogenic mechanism for the EMT in high fructose-induced liver fibrosis. Fructose feeding induces insulin resistance and the aberrant expression of miRNAs in mouse livers [51]. Changes in miRNA expression have been reported during all stages of diabetes and other metabolic disorders, [52] and lipid and carbohydrate metabolic pathways in high fructose-fed mouse livers [51]. These miRNAs assemble as a regulatory network that cooperatively targets the lipid metabolic pathway and insulin signaling at multiple levels to amplify their regulatory effects on target genes and contribute to the pathogenesis of high fructose-induced insulin resistance [51]. Obesity and insulin resistance are strongly associated with nonalcoholic fatty liver disease and possibly the development of liver fibrosis $[53,54]$. Therefore, the high fructose-induced miR375-3p downregulation may be related to the lipid metabolic pathway and insulin signaling disorder. Further studies are needed to identify the miR-375-3p-triggered profibrogenic molecules that evoke liver fibrosis. 
a

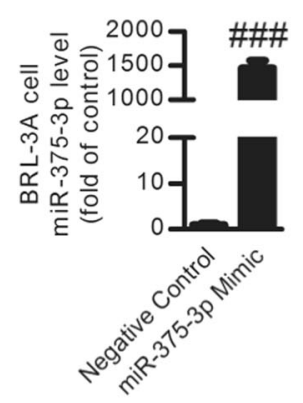

JAK2 $=125 \mathrm{kDa}$

$37 \mathrm{kDa}$

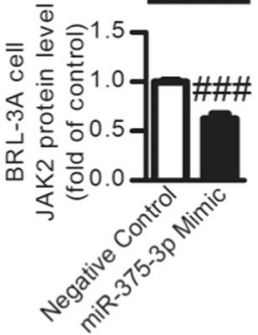

C

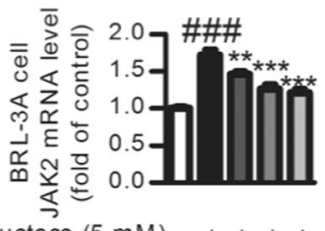

Fructose $(5 \mathrm{mM})-++++$ Negative control $(50 \mathrm{nM})++\ldots$ miR-375-3p Mimic $(50 \mathrm{nM})-\cdots++$ Magnesium $(40 \mu \mathrm{M})$ - . - +

Pioglitazone $(10 \mu \mathrm{M})$ - - - + d

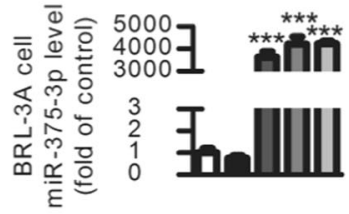

Fructose $(5 \mathrm{mM})-++++$ Negative control $(50 \mathrm{nM})++\ldots$ miR-375-3p Mimic (50 nM) - - + + + isoglycyrnizinate $(40 \mu \mathrm{M})$ - - - + Pioglitazone $(10 \mu \mathrm{M})$ - - - + + e
Negative-Control (50 nM)
Fructose $(5 \mathrm{mM})+$ Negative-Control $(50 \mathrm{nM})$
Fructose $(5 \mathrm{mM})+\operatorname{miR}-375-3 p$ Mimic $(50 \mathrm{nM})$
Fructose $(5 \mathrm{mM})+\operatorname{miR}-375-3 p$ Mimic $(50 \mathrm{nM})$
+ Magnesium isoglycyrrhizinate $(40 \mu \mathrm{M})$
Fructose $(5 \mathrm{mM})+\operatorname{miR}-375-3 p$ Mimic $(50 \mathrm{nM})$
+ Pioglitazone $(10 \mu \mathrm{M})$

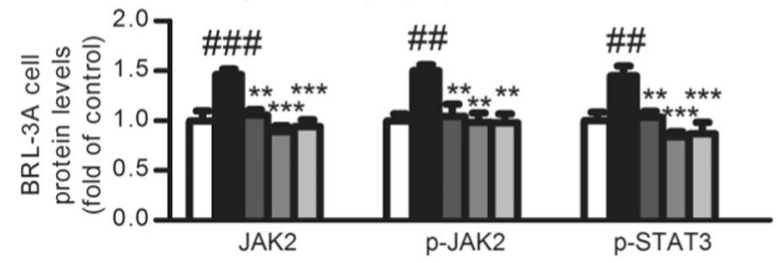

f

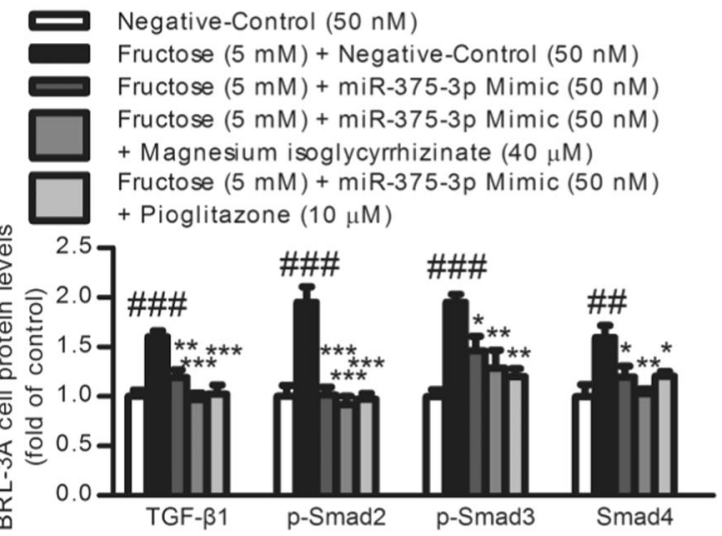

g

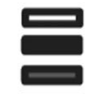

Negative-Control $(50 \mathrm{nM})$

Fructose $(5 \mathrm{mM})+$ Negative-Control $(50 \mathrm{nM})$

Fructose $(5 \mathrm{mM})+\operatorname{miR}-375-3 p$ Mimic $(50 \mathrm{nM})$

Fructose $(5 \mathrm{mM})+\operatorname{miR}-375-3 p$ Mimic (50 $\mathrm{nM}$ )

+ Magnesium isoglycyrrhizinate $(40 \mu \mathrm{M})$

Fructose $(5 \mathrm{mM})+\mathrm{miR}-375-3 p \mathrm{Mimic}(50 \mathrm{nM})$

+ Pioglitazone $(10 \mu \mathrm{M})$

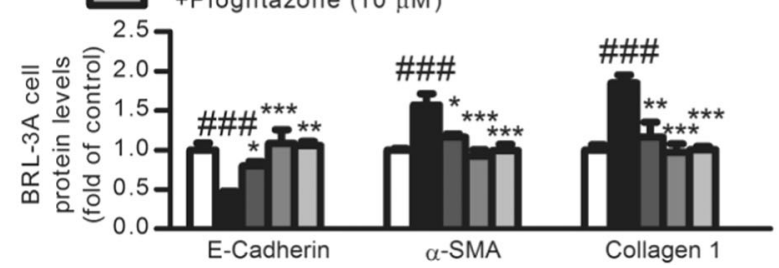

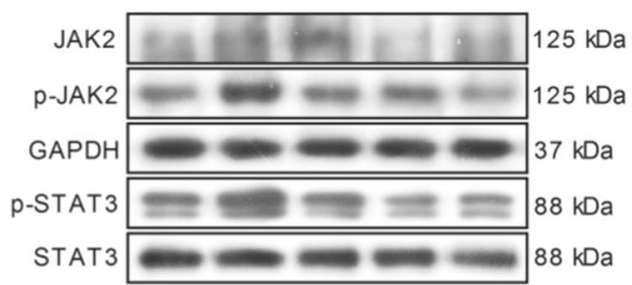

Fructose $(5 \mathrm{mM})$

Negative-Control $(50 \mathrm{nM})$

miR-375-3p Mimic (50 nM)

$\underset{\text { Magnesium }}{\text { Maglycyrrhizinate }}(40 \mu \mathrm{M})$

Pioglitazone $(10 \mu \mathrm{M})$
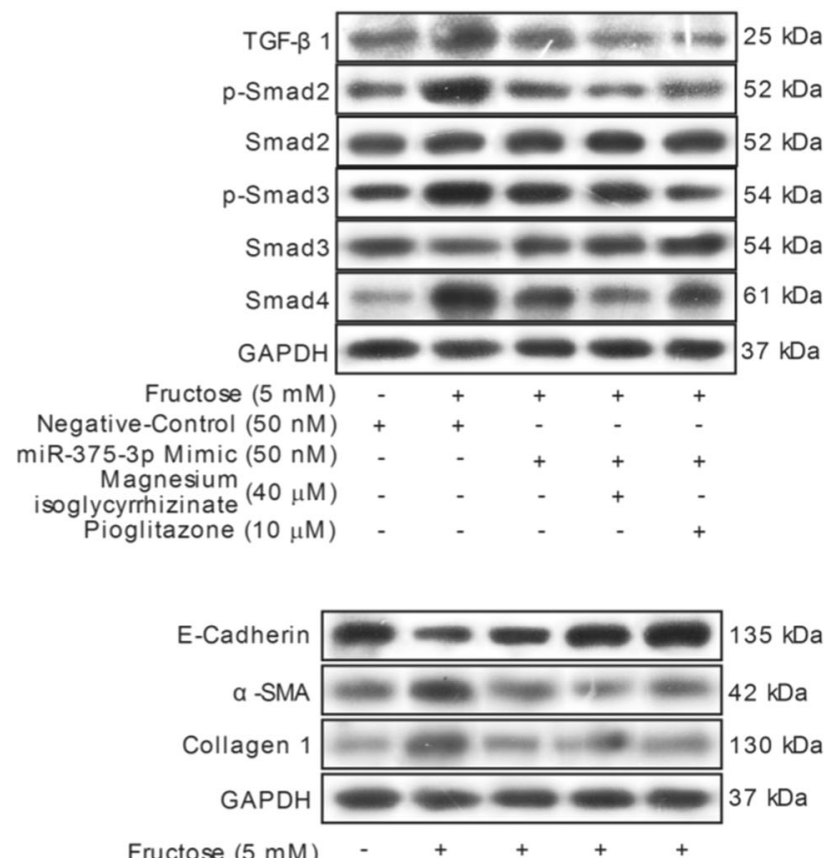

Negative-Control (50 nM)

miR-375-3p Mimic (50 nM)

Magnesium $(40 \mu \mathrm{M})$

Pioglitazone $(10 \mu \mathrm{M})$

Fig. 8 Magnesium isoglycyrrhizinate upregulates miR-375-3p to partially prevent the JAK2/STAT3 pathway and TGF- $\beta 1 / \mathrm{Smads}$ signaling activation in fructose-exposed BRL-3A cells. a Transfection efficiency in BRL-3A cells transfected with the miR-375-3p mimic (50 nM) or negative control was evaluated by a qRT-PCR analysis and normalized to U6 expression $(n=3)$. Relative cell lysate JAK2 protein levels (b), JAK2 mRNA levels (c), miR-375-3p expression (d), and JAK2, p-JAK2, p-STAT3, TGF- $\beta 1$, p-Smad2, p-Smad3, Smad4, E-Cadherin, $\alpha$-SMA, and Collagen 1 protein levels $(\mathbf{e}-\mathbf{g})$ in miR-375-3p mimic-transfected BRL-3A cells were determined by a qRT-PCR analysis or Western blot analysis. Relative miR-375-3 levels were normalized to U6 expression $(n=6)$. Relative protein levels of JAK2, p-JAK2, TGF- $\beta 1$, Smad4, E-Cadherin, $\alpha$-SMA, and Collagen 1 were normalized to GAPDH, and those of p-STAT3, p-Smad2, and p-Smad3 were normalized to STAT3, Smad2, and Smad3, respectively $(n=6)$. The data are expressed as the mean \pm SEM. ${ }^{\# \#} P<0.01,{ }^{\# \# \#} P<0.001$ versus Normal-Control; ${ }^{*} P<0.05,{ }^{* * *} P<0.01,{ }^{* * *} P<0.001$ versus Fructose-Vehicle or Fructose-Vehicle + miR-375-3p mimic negative control 

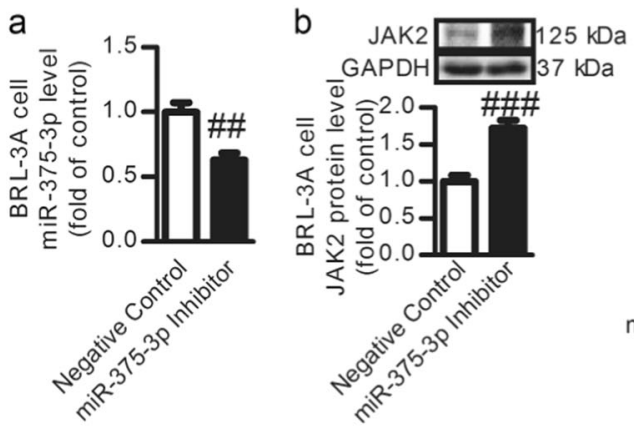

C

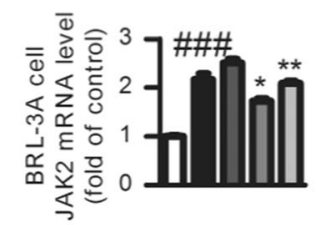

Fructose $(5 \mathrm{mM})-++++$ Negative control $(80 \mathrm{nM})++\ldots$ miR-375-3p Inhibitor (80 nM) - - + + + isoglycyrrhizinate $(40 \mu \mathrm{M}) \cdots \cdot+$

Pioglitazone $(10 \mu \mathrm{M})$ - - - - + d

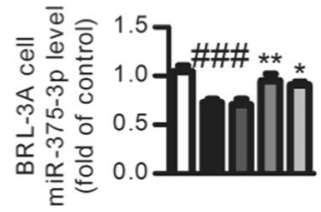

Fructose $(5 \mathrm{mM})-++++$ Negative control $(80 \mathrm{nM})++\ldots$ miR-375-3p Inhibitor (80 nM) - - + + + Magnesium
isoglycyrnhizinate $(40 \mu \mathrm{M})$ - - - + Pioglitazone $(10 \mu \mathrm{M})$ - - - + e

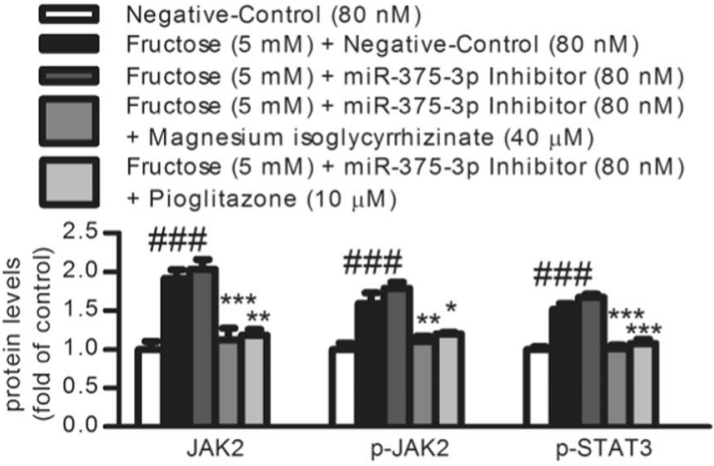

f

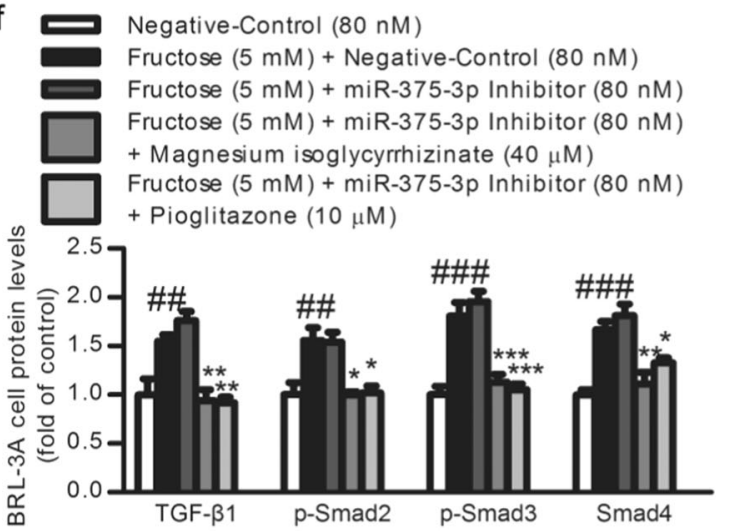

$\mathrm{g} \rightleftharpoons$ Negative-Control $(80 \mathrm{nM})$

Fructose $(5 \mathrm{mM})+$ Negative-Control $(80 \mathrm{nM})$

Fructose $(5 \mathrm{mM})+$ miR-375-3p Inhibitor $(80 \mathrm{nM})$

Fructose $(5 \mathrm{mM})+\mathrm{miR}-375-3 p$ Inhibitor $(80 \mathrm{nM})$

+ Magnesium isoglycyrrhizinate $(40 \mu \mathrm{M})$

Fructose $(5 \mathrm{mM})+\mathrm{miR}-375-3 \mathrm{p}$ Inhibitor $(80 \mathrm{nM})$

+ Pioglitazone $(10 \mu \mathrm{M})$

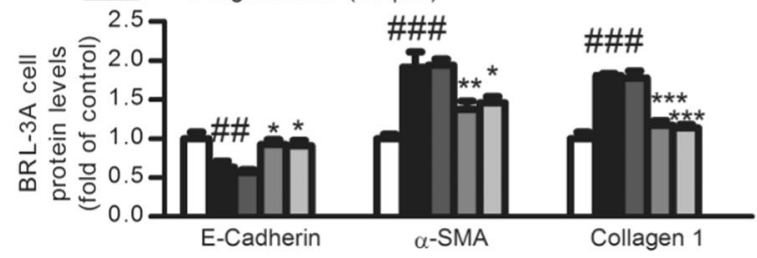

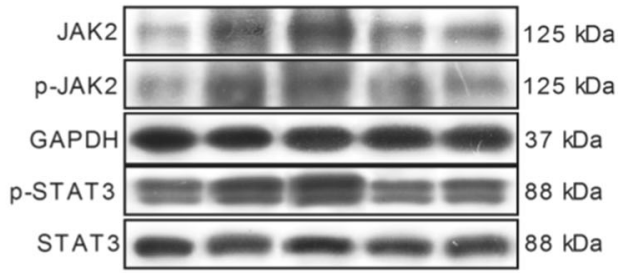

Fructose $(5 \mathrm{mM})$

Negative-Control $(80 \mathrm{nM})$

miR-375-3p Inhibitor ( $80 \mathrm{nM}$ ) Magnesium

isoglycyrrhizinate $(40 \mu \mathrm{M})$

Pioglitazone $(10 \mu \mathrm{M})$

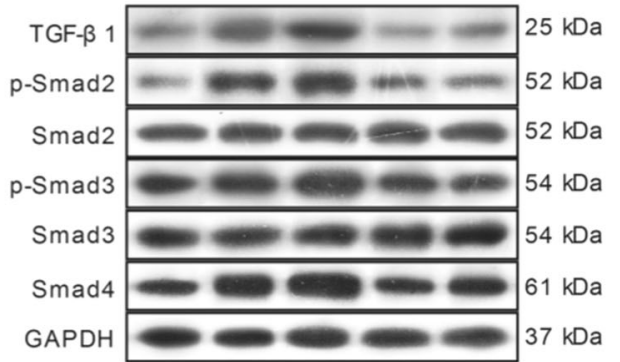

Fructose $(5 \mathrm{mM})$ Negative-Control $(80 \mathrm{nM})$ miR-375-3p Inhibitor ( $80 \mathrm{nM})$ $\underset{\text { Magnesium }}{\text { Magoglycyrrhizinate }}(40 \mu \mathrm{M})$ Pioglitazone $(10 \mu \mathrm{M})$

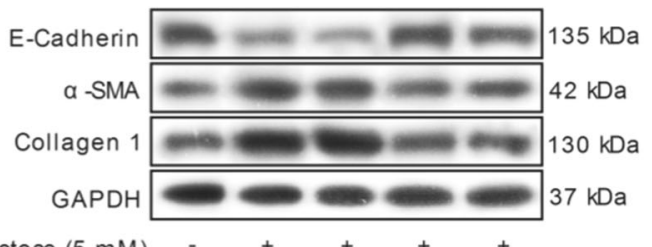

Fructose $(5 \mathrm{mM})$

Negative-Control $(80 \mathrm{nM})$

miR-375-3p Inhibitor ( $80 \mathrm{nM}$ ) Magnesium isoglycyrrhizinate

Pioglitazone $(10 \mu \mathrm{M})$

Fig. 9 Magnesium isoglycyrrhizinate inhibits low miR-375-3p expression to partially prevent the JAK2/STAT3 pathway and TGF- $\beta 1 / \mathrm{Smads}$ signaling activation in fructose-exposed BRL-3A cells. a Transfection efficiency in BRL-3A cells transfected with the miR-375-3p inhibitor $(80 \mathrm{nM})$ or negative control was evaluated by a qRT-PCR analysis and normalized to U6 expression $(n=3)$. Relative cell lysate JAK2 protein levels (b), JAK2 mRNA levels (c), miR-375-3p expression (d), and JAK2, p-JAK2, p-STAT3, TGF- $\beta 1$, p-Smad2, p-Smad3, Smad4, E-Cadherin, $\alpha$-SMA, and Collagen 1 protein levels (e-g) in miR-375-3p inhibitor-transfected BRL-3A cells were determined by qRT-PCR or a Western blot analysis. Relative miR-375-3p levels were normalized to U6 expression $(n=6)$. Relative protein levels of JAK2, p-JAK2, TGF- $\beta 1$, Smad4, E-Cadherin, $\alpha$ SMA, and Collagen 1 were normalized to GAPDH, and those of p-STAT3, p-Smad2, and p-Smad3 were normalized to STAT3, Smad2, and

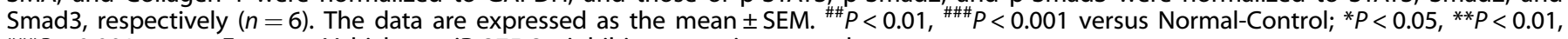
${ }^{* * *} P<0.001$ versus Fructose-Vehicle + miR-375-3p inhibitor negative control 


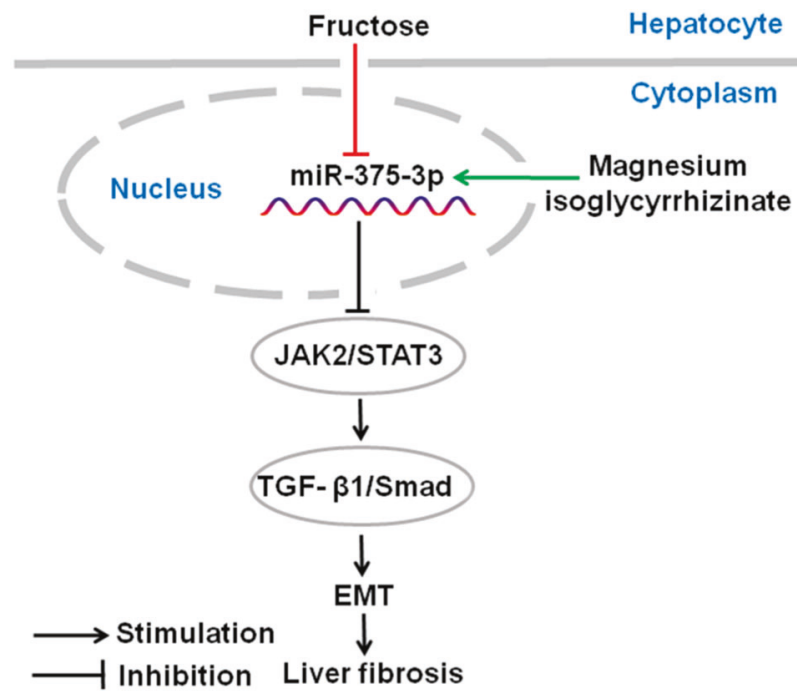

Fig. 10 The hypothetical mechanisms by magnesium isoglycyrrhizinate which prevents fructose-induced hepatocyte EMT in liver fibrosis. Fructose down-regulates miR-375-3p expression to activate JAK2/STAT3 pathway and TGF- $\beta 1 /$ Smad signaling, promoting hepatocyte EMT in liver fibrosis. Magnesium isoglycyrrhizinate protects against high fructose-induced EMT and liver fibrosis by increasing miR-375-3p expression to inhibit the JAK2/STAT3 pathway and then restrain TGF- $\beta 1 /$ Smad signaling activation

Magnesium isoglycyrrhizinate has been reported to decrease the STAT3 protein levels and protect remnant liver function in rats with $90 \%$ liver resection, [55] reduce the TGF- $\beta 1$ and $p$-Smad3 protein levels, and alleviate carbon tetrachloride-induced liver fibrosis in rats. ${ }^{28}$ Here, we found that magnesium isoglycyrrhizinate inhibited the high fructose-induced JAK2/STAT3 pathway in rat livers and hepatocytes. This blockade by magnesium isoglycyrrhizinate suppressed TGF- $\beta 1 / \mathrm{Smads}$ signaling activation, which is consistent with its alleviation of high fructose-induced EMT. Furthermore, magnesium isoglycyrrhizinate was found to increase hepatocyte miR-375-3p expression in animal and cell models. The antifibrotic role of magnesium isoglycyrrhizinate may be at least partially due to high miR-375-3p expression-triggered inhibition of the JAK2/STAT3 pathway and TGF- $\beta 1 /$ Smads signaling activation, resulting in the attenuation of the EMT following high fructose induction. MiR-375-3p in hepatocytes may be considered a reversible switch, highlighting the possibility of using magnesium isoglycyrrhizinate to treat high fructose-induced liver fibrosis. Further studies should investigate the role of magnesium isoglycyrrhizinate in modulating the physiology and pathology of miR-375-3p in the EMT in liver fibrosis.

In conclusion, this study for the first time demonstrates that magnesium isoglycyrrhizinate protects against high fructoseinduced EMT and liver fibrosis by increasing miR-375-3p expression to inhibit the JAK2/STAT3 pathway and then restrain TGF- $\beta 1 /$ Smads signaling activation. These results provide a potential novel strategy for the prevention and treatment of liver fibrosis associated with excess fructose intake using magnesium isoglycyrrhizinate.

\section{ACKNOWLEDGEMENTS}

This work was partially supported by the Natural Science Foundation of Jiangsu Province (Grant No. BK20141243).

\section{AUTHOR CONTRIBUTIONS}

Conducted the experiments: YZY, XJZ, YP. Contributed new reagents or analytic tools: YP, SCW and SJW. Performed the data analysis: YZY, XJZ, LDK, QXU, HMG, and HJX. Wrote or contributed to the writing of the manuscript: LDK, YZY, QX, HJX, and RQJ. Participated in the research design: LDK and HMG

\section{REFERENCES}

1. Yu K, Li Q, Shi G, Li N. Involvement of epithelial-mesenchymal transition in liver fibrosis. Saudi J Gastroenterol. 2018;24:5-11.

2. Park JH, Park B, Park KK. Suppression of hepatic epithelial-to-mesenchymal transition by melittin via blocking of TGFbeta/Smad and MAPK-JNK signaling pathways. Toxins (Basel). 2017;9:138-152.

3. Park JH, Yoon J, Lee KY, Park B. Effects of geniposide on hepatocytes undergoing epithelial-mesenchymal transition in hepatic fibrosis by targeting TGFbeta/Smad and ERK-MAPK signaling pathways. Biochimie. 2015;113:26-34.

4. Granzow M, Schierwagen R, Klein S, Kowallick B, Huss S, Linhart M, et al. Angiotensin-II type 1 receptor-mediated Janus kinase 2 activation induces liver fibrosis. Hepatology. 2014;60:334-348.

5. Lu C, Xu W, Shao J, Zhang F, Chen A, Zheng S. Nrf2 induces lipocyte phenotype via a SOCS3-dependent negative feedback loop on JAK2/STAT3 signaling in hepatic stellate cells. Int Immunopharmacol. 2017;49:203-211.

6. Ogata $\mathrm{H}$, Chinen T, Yoshida T, Kinjyo I, Takaesu G, Shiraishi H, et al. Loss of SOCS3 in the liver promotes fibrosis by enhancing STAT3-mediated TGF-beta1 production. Oncogene. 2006;25:2520-2530.

7. Cydylo MA, Davis AT, Kavanagh K. Fatty liver promotes fibrosis in monkeys consuming high fructose. Obesity. 2017;25:290-293.

8. Ichimura M, Masuzumi M, Kawase M, Sakaki M, Tamaru S, Nagata Y, Tanaka K, et al. A diet-induced Sprague-Dawley rat model of nonalcoholic steatohepatitisrelated cirrhosis. J Nutr Biochem. 2016;40:62-69.

9. Zhou GY, Yi YX, Jin LX, Lin W, Fang PP, Lin XZ, et al. The protective effect of juglanin on fructose-induced hepatitis by inhibiting inflammation and apoptosis through TLR4 and JAK2/STAT3 signaling pathways in fructose-fed rats. Biomed Pharmacother. 2016;81:318-328.

10. Tu $X$, Zhang $H$, Zhang J, Zhao S, Zheng $X$, Zhang Z, et al. MicroRNA-101 suppresses liver fibrosis by targeting the TGFbeta signalling pathway. J Pathol. 2014;234:46-59.

11. Szabo G, Bala S. MicroRNAs in liver disease. Nat Rev Gastroenterol Hepatol. 2013;10:542-552

12. Wang XW, Heegaard NH, Orum H. MicroRNAs in liver disease. Gastroenterology. 2012;142:1431-1443.

13. Pottier N, Cauffiez C, Perrais M, Barbry P, Mari B. FibromiRs: translating molecular discoveries into new anti-fibrotic drugs. Trends Pharmacol Sci. 2014;35:119-126.

14. Guo $Y$, Xiong $Y$, Sheng $Q$, Zhao S, Wattacheril J, Flynn CR. A micro-RNA expression signature for human NAFLD progression. J Gastroenterol. 2016;51:1022-1030.

15. Yin LH, Zheng XQ, Li HY, Bi LX, Shi YF, Ye AF, et al. Epigenetic deregulated miR375 contributes to the constitutive activation of JAK2/STAT signaling in myeloproliferative neoplasm. Leuk Res. 2015;39:471-478.

16. Xu Y, Jin J, Liu Y, Huang Z, Deng Y, You T, et al. Snail-regulated miR-375 inhibits migration and invasion of gastric cancer cells by targeting JAK2. PLoS One. 2014;9:e99516.

17. Ye F, Tang C, Shi W, Qian J, Xiao S, Gu M, et al. A MDM2-dependent positivefeedback loop is involved in inhibition of miR-375 and miR-106b induced by Helicobacter pylori lipopolysaccharide. Int J Cancer. 2015;136:2120-2131.

18. Lamouille $S, X u$ J, Derynck R. Molecular mechanisms of epithelial-mesenchymal transition. Nat Rev Mol Cell Biol. 2014;15:178-6.

19. Kawaguchi-Suzuki M, Bril F, Kalavalapalli S, Cusi K, Frye RF. Concentrationdependent response to pioglitazone in nonalcoholic steatohepatitis. Aliment Pharmacol Ther. 2017;46:56-61.

20. Chan WK, Treeprasertsuk S, Imajo K, Nakajima A, Seki Y, Kasama K, et al. Clinical features and treatment of nonalcoholic fatty liver disease across the Asia Pacific region-the GO ASIA initiative. Aliment Pharmacol Ther. 2018;47:816-825.

21. Garry EM, Buse JB, Lund JL, Pate V, Sturmer T. Comparative safety of pioglitazone versus clinically meaningful treatment alternatives concerning the risk of bladder cancer in older US adults with type 2 diabetes. Diabetes Obes Metab. 2018;20:129-140.

22. Yan $Y$, Mo $Y$, Zhang $D$. Magnesium isoglycyrrhizinate prevention of chemotherapy-induced liver damage during initial treatment of patients with gastrointestinal tumors. Chin J Hepatol. 2015;23:204-208.

23. Chen KJ, Chen WY, Chen X, Jia YM, Peng GQ, Chen L, et al. Increased elimination of paclitaxel by magnesium isoglycyrrhizinate in epithelial ovarian cancer patients treated with paclitaxel plus cisplatin: a pilot clinical study. Eur J Drug Metab Pharmacokinet. 2014;39:25-31. 
24. Vincenzi B, Armento G, Spalato Ceruso M, Catania G, Leakos M, Santini D, et al. Drug-induced hepatotoxicity in cancer patients-implication for treatment. Expert Opin Drug Saf. 2016;15:1219-1238.

25. Lv J, Xiao Q, Chen Y, Fan X, Liu X, Liu F, et al. Effects of magnesium isoglycyrrhizinate on AST, ALT, and serum levels of Th1 cytokines in patients with alloHSCT. Int Immunopharmacol. 2017;46:56-61.

26. Li SL, Cui XL, Ji WY, Lin J. Clinical effect on the treatment of acute drug-induced liver injury with magnesium isoglycyrrhizinate. Chin J Coal Ind Med. 2014;17:368-370.

27. Cui HS, Chen N. The effect of magnesium isoglycyrrhizinate on indicators of hepatic fibrosis in patients with chronic type B hepatitis. World Clin Drugs. 2008;29:670-673.

28. Peng ZT, Li J, Wang P, Li HM, Liu SX. Effects of magnesium isoglycyrrhizinate on hepatic TGF- $\beta 1$ and Smad protein expression in rats with experimental hepatic fibrosis. Prog Mod Biomed. 2011;11:240-242.

29. Bian $M$, Chen $X$, Zhang $C$, Jin $H$, Wang $F$, Shao J, et al. Magnesium isoglycyrrhizinate promotes the activated hepatic stellate cells apoptosis via endoplasmic reticulum stress and ameliorates fibrogenesis in vitro and in vivo. Biofactors. 2017;43:836-846.

30. Zhao XJ, Yang YZ, Zheng YJ, Wang SC, Gu HM, Pan Y, et al. Magnesium isoglycyrrhizinate blocks fructose-induced hepatic NF-kappaB/NLRP3 inflammasome activation and lipid metabolism disorder. Eur J Pharmacol. 2017;809:141-150.

31. Zhang JC, Zheng GF, Wu MX, Wu JW, Ouyang LY, Liu XQ. Effect of magnesium isoglycyrrhizinate on PLA2 during liver tissue injury following limb ischemia/ reperfusion in rats. Chin J Hepatol. 2012;20:537-541.

32. Mao YM, Zeng MD, Chen Y, Chen CW, Fu QC, Cai X, et al. Magnesium isoglycyrrhizinate in the treatment of chronic liver diseases: a randomized, doubleblind, multi-doses, active drug controlled, multi-center study. Chin J Hepatol. 2009;17:847-851.

33. Qu B, Xing R, Wang $H$, Chen $X, G e ~ Q$, Peng D, et al. Multiple effects of magnesium isoglycyrrhizinate on the disposition of docetaxel in docetaxel-induced liver injury. Xenobiotica. 2017;47:290-296.

34. Sun L, Shen J, Pang X, Lu L, Mao Y, Zeng M. Phase I safety and pharmacokinetic study of magnesium isoglycyrrhizinate after single and multiple intravenous doses in chinese healthy volunteers. J Clin Pharmacol. 2007;47:767-773.

35. Shen L, Hillebrand A, Wang DQ, Liu M. Isolation and primary culture of rat hepatic cells. J Vis Exp. 2012;64:e3917.

36. Huang X, Qin J, Lu S. Magnesium isoglycyrrhizinate protects hepatic L02 cells from ischemia/reperfusion induced injury. Int J Clin Exp Pathol. 2014;7:4755.

37. Yoshikawa H, Matsubara K, Qian GS, Jackson P, Groopman JD, Manning JE, et al. SOCS-1, a negative regulator of the JAK/STAT pathway, is silenced by methylation in human hepatocellular carcinoma and shows growth-suppression activity. Nat Genet. 2001;28:29-35.

38. Wang Y, Hospital LC. Clinical curative effect of magnesium isoglycyrrhizinate in treatment of chronic liver disease and its influence on improvement of laboratory indexes. Chin Med Pharm. 2016;6:82-84.
39. Alisi A, Manco M, Pezzullo M, Nobili V. Fructose at the center of necroinflammation and fibrosis in nonalcoholic steatohepatitis. Hepatology. 2011;53:372-373.

40. Vos MB, Lavine JE. Dietary fructose in nonalcoholic fatty liver disease. Hepatology. 2013:57:2525-2531.

41. McCommis KS, Hodges WT, Brunt EM, Nalbantoglu I, McDonald WG, Holley C, et al. Targeting the mitochondrial pyruvate carrier attenuates fibrosis in a mouse model of nonalcoholic steatohepatitis. Hepatology. 2017;65:1543-1556.

42. Chen YL, Lv J, Ye XL, Sun MY, Xu Q, Liu CH, et al. Sorafenib inhibits transforming growth factor beta1-mediated epithelial-mesenchymal transition and apoptosis in mouse hepatocytes. Hepatology. 2011;53:1708-1718.

43. Zeisberg M, Yang C, Martino M, Duncan MB, Rieder F, Tanjore $H$, et al. Fibroblasts derive from hepatocytes in liver fibrosis via epithelial to mesenchymal transition. J Biol Chem. 2007;282:23337-23347.

44. Wu KM, Ye C, Lin L, Chu Y, Ji M, Dai W, et al. Inhibiting miR-21 attenuates experimental hepatic fibrosis by suppressing both the ERK1 pathway in HSC and hepatocyte EMT. Clin Sci (Lond). 2016;130:1469-1480.

45. Kong D, Zhang F, Shao J, Wu L, Zhang $X$, Chen L, et al. Curcumin inhibits cobalt chloride-induced epithelial-to-mesenchymal transition associated with interference with TGF-beta/Smad signaling in hepatocytes. Lab Invest. 2015;95:1234-1245.

46. Zhao S, Zhang Y, Zheng X, Tu X, Li H, Chen J, et al. Loss of microRNA-101 promotes epithelial to mesenchymal transition in hepatocytes. J Cell Physiol. 2015;230:2706-2717.

47. Seki E, Schwabe RF. Hepatic inflammation and fibrosis: functional links and key pathways. Hepatology. 2015;61:1066-1079.

48. Tsuchida T, Friedman SL. Mechanisms of hepatic stellate cell activation. Nat Rev Gastroenterol Hepatol. 2017;14:397-411.

49. Taura K, Miura K, Iwaisako K, Osterreicher CH, Kodama Y, Penz-Osterreicher M, et al. Hepatocytes do not undergo epithelial-mesenchymal transition in liver fibrosis in mice. Hepatology. 2010;51:1027-1036.

50. Starsíchová A, Lincová E, Pernicová Z, Kozubík A, Soucek K. TGF-beta1 suppresses IL-6-induced STAT3 activation through regulation of Jak2 expression in prostate epithelial cells. Cell Signal. 2010;22:1734-1744.

51. Sud N, Zhang H, Pan K, Cheng X, Cui J, Su Q, et al. Aberrant expression of microRNA induced by high-fructose diet: implications in the pathogenesis of hyperlipidemia and hepatic insulin resistance. J Nutr Biochem. 2017;43:125-131.

52. Pandey AK, Agarwal P, Kaur K, Datta M. MicroRNAs in diabetes: tiny players in big disease. Cell Physiol Biochem. 2009;23:221-232.

53. Rinella ME. Nonalcoholic fatty liver disease: a systematic review. JAMA. 2015:313:2263-2273.

54. Hardy T, Oakley F, Anstee QM, Day CP. Nonalcoholic fatty liver disease: pathogenesis and disease spectrum. Annu Rev Pathol. 2016;11:451-496.

55. Tang GH, Yang HY, Zhang JC, Ren JJ, Sang XT, Lu X, et al. Magnesium isoglycyrrhizinate inhibits inflammatory response through STAT3 pathway to protect remnant liver function. World J Gastroenterol. 2015;21:12370-12380. 